ESAIM: M2AN 50 (2016) 851-877

DOI: $10.1051 / \mathrm{m} 2 \mathrm{an} / 2015088$
ESAIM: Mathematical Modelling and Numerical Analysis

www.esaim-m2an.org

\title{
THE ARBITRARY ORDER MIXED MIMETIC FINITE DIFFERENCE METHOD FOR THE DIFFUSION EQUATION
}

\author{
Vitaliy Gyrya ${ }^{1}$, Konstantin Lipnikov ${ }^{1}$ and Gianmarco ManZini ${ }^{1,2,3}$
}

\begin{abstract}
We propose an arbitrary-order accurate mimetic finite difference (MFD) method for the approximation of diffusion problems in mixed form on unstructured polygonal and polyhedral meshes. As usual in the mimetic numerical technology, the method satisfies local consistency and stability conditions, which determines the accuracy and the well-posedness of the resulting approximation. The method also requires the definition of a high-order discrete divergence operator that is the discrete analog of the divergence operator and is acting on the degrees of freedom. The new family of mimetic methods is proved theoretically to be convergent and optimal error estimates for flux and scalar variable are derived from the convergence analysis. A numerical experiment confirms the high-order accuracy of the method in solving diffusion problems with variable diffusion tensor. It is worth mentioning that the approximation of the scalar variable presents a superconvergence effect.
\end{abstract}

Mathematics Subject Classification. 65N30, 65N12, 65G99, 76R99.

Received April 19, 2015. Revised October 15, 2015.

Published online May 23, 2016.

\section{INTRODUCTION}

In the last decade, there has been a huge amount of work aimed at designing new discretization methods for PDEs that work on unstructured polygonal and polyhedral meshes. General unstructured meshes appear in various engineering applications, either as a flexible way to capture complex geometries (modeling of fluid flows in porous medium) or as a product of mesh optimization algorithms (mesh reconnection in arbitrarily LagrangianEulerian methods). Major challenges for numerical solution of PDEs on general meshes are simplicity of extension of two dimensional schemes to three dimensions and to arbitrarily order of accuracy.

Successful discretization methods on general meshes were developed using both finite volume (FV) [32] and finite element (FE) frameworks $[15,16]$. FV methods are natural for general meshes but historically, they were low-order methods. Examples of FV methods for elliptic PDEs include the multi-point flux approximation (MPFA) method [1,2], the hybrid FV method [33] (the resulting scheme is also know as SUSHI), the discrete duality finite volume (DDFV) method [24,28,35], the gradient method [31]; see also the recent review [29].

\footnotetext{
Keywords and phrases. Mimetic finite difference method, polygonal mesh, high-order discretization, Poisson problem, mixed formulation.

1 Los Alamos National Laboratory, Theoretical Division, Group T-5, MS B284, Los Alamos, NM-87545, USA. gyrya@lanl.gov; lipnikov@lanl.gov; gmanzini@lanl.gov

2 Istituto di Matematica Applicata e Tecnologie Informatiche, Consiglio Nazionale delle Ricerche (IMATI-CNR), via Ferrata 1, 27100 Pavia, Italy. gm.manzini@gmail.com

3 Centro di Simulazione Numerica Avanzata (CeSNA) - IUSS Pavia, v.le Lungo Ticino Sforza 56, 27100 Pavia, Italy.
} 
Extension of FV methods to higher order is done either by extending the discretization stencil or by enriching the local approximation space, in a spirit of the FE method. For the elliptic PDEs, symmetry and positive definiteness of the resulting matrices are the properties that are difficult to achieve for higher-order schemes.

On the other hand, the conventional arbitrarily order FE method on simplicial meshes preserves all fundamental properties of elliptic PDEs. However, its extension to general meshes is a work in progress that is based on the new idea of the virtual finite element space [5]. Other FE methods that work on general meshes include the polygonal/polyhedral finite element method (PFEM) [46,49-51], hybrid high-order method [26, 27], the discontinuous Galerkin (DG) method [25], hybridized discontinuous Galerkin (HDG) method [23], and the weak Galerkin (wG) method [38, 52, 53].

In between these two general frameworks, we find the mimetic finite difference (MFD) method $[11,14,41,48]$ which combines best properties of both frameworks. Similar to FV methods, the MFD method imposes no critical constraints on the computational mesh which may contain all types of polygonal and polyhedral elements, including non-convex elements. Similar to FE methods, a duality argument is the essential part of the MFD construction which implies symmetry and positivity of matrices for schemes of any order of accuracy. Construction of stiffness and mass matrices is unique for the mimetic framework and is based on the stability and local consistency conditions. A by-product of these conditions is that there exists a rich family of schemes with similar approximation properties. This remains true for mimetic schemes of simplicial meshes. In many cases this family contains schemes with additional properties such as the discrete maximum principle [42].

In recent years, the MFD method has been extensively developed for the solution of a wide range of scientific and engineering problems in continuum mechanics [47], discretization of differential forms [14, 18, 48], electromagnetics [36,40], diffusion [37], convection-diffusion [6], steady Stokes [7,10], elasticity [4], elliptic obstacles [3], Reissner-Mindlin plates [13], eigenvalues [21], and two-phase flows in porous media [44]. An extensive list of references can be found in book [11] and review paper [41]. Connection of low-order MFD schemes for elliptic problems with two FV schemes has been established in [6,30].

The MFD method considered in the aforementioned applications typically provides a low-order approximation of the unknowns with, at the best, the second-order convergence for scalar unknowns. An open issue in the development of the mimetic technology is the construction of high-order schemes, which is still a challenging task even for two-dimensional and three-dimensional second-order elliptic problems. For pure diffusion problems in primal form, the mimetic method in [9] is the extension to arbitrary order of accuracy of the low-order method proposed in [17]. However, for pure diffusion problems in mixed form, a high-order accurate formulation is still missing in the literature, although a first attempt to this direction was done in $[8,12,34]$. In these papers, the authors present an improvement from first- to second-order accurate discretization of the flux unknown of the MFD method in [19]. A second major issue, which impacts also the other methods mentioned above, is the treatment of non-constant coefficients like a varying diffusion tensor while preserving the high-order of accuracy of the approximation. To this end, we apply the technique that was proved successful in $[8,39]$ for both the mixed low-order method and the high-order primal formulation in two- and three-dimensional elliptic problems.

The goals of this work are (a) to develop a new family of arbitrary-order mimetic schemes for mixed formulation of the elliptic PDE; (b) to present a solid theoretical framework where the convergence of the method is proved and error estimates are derived; (c) to investigate the performance of the method numerically by solving diffusion problems with a smoothly variable full tensor diffusion coefficient on different types of unstructured polygonal meshes.

Flexibility on the MFD framework for selecting non-standard degrees of freedom and non-standard approximations of primary operators allows us to develop and analyze mimetic schemes tailored for particular applications such as the Lagrangian gasdynamics [20,41] and nonlinear elliptic PDEs with degenerate coefficients [43].

The approximation of the scalar unknown and the flux is seeked in finite dimensional linear spaces of grid functions whose definition relies on polynomial moments of degree $k \geq 0$ inside each cell and of degree $k+1$ on each cell interface. We equip these finite dimensional linear spaces with suitable mimetic inner products, whose construction is detailed in the paper. Error estimates are derived by using the mesh dependent norms induced by these inner products and assuming that the scalar unknown is in $H^{k+3}(\Omega)$. These result is in agreement 
with the analysis of the method considered in [8], which coincide with the method presented in this paper for $k=0$, where the scalar unknown was assumed in $H^{3}(\Omega)$.

In this work we also present a novel ultraconvergence phenomenon that we think is related to the special selection of the degrees of freedom. It was discovered in our numerical experiments and still needs a theoretical explanation.

The paper outline is as follows. The formulation of the MFD method is presented in Section 2. Preliminary results on the approximation properties of interpolation and projection operators are reviewed in Section 3. The convergence analysis is carried out in Section 4 for the flux variable and in Section 5 for the scalar variable. The expected convergence rates are confirmed with the numerical experiments in Section 6. Final remarks and conclusions are given in Section 7.

\section{Mimetic Finite Difference Formulation}

Let $\Omega \subset \mathbb{R}^{d}$ be a polygonal domain for $d=2$ or a polyhedral domain for $d=3$ with Lipschitz boundary $\Gamma$. We consider the mimetic approximation of the steady diffusion problem in mixed form for the scalar variable $p$ and its vector flux $\mathbf{u}$ :

$$
\begin{aligned}
\mathbf{u} & =-\mathrm{K} \nabla p & & \text { in } \Omega, \\
\operatorname{div} \mathbf{u} & =f & & \text { in } \Omega, \\
p & =g & & \text { on } \Gamma .
\end{aligned}
$$

The functions $f$ and $g$ are, respectively, the source term and the boundary data and $\mathrm{K}$ is a full symmetric tensor describing the material properties.

We set the mixed variational formulation using the functional space $L^{2}(\Omega)$ for the scalar variable and $H(\operatorname{div}, \Omega)$ for the flux variable. Here, $L^{2}(\Omega)$ is the standard space of square integrable functions defined on $\Omega$, and $H(\operatorname{div}, \Omega)$ is the Sobolev space of vector-valued functions in $\left(L^{2}(\Omega)\right)^{d}$ with square integrable divergence.

The mixed variational formulation of problems (2.1a)-(2.1c) reads as [15]:

find $(\mathbf{u}, p) \in H(\operatorname{div}, \Omega) \times L^{2}(\Omega)$ such that

$$
\begin{aligned}
\left(\mathrm{K}^{-1} \mathbf{u}, \mathbf{v}\right)-(p, \operatorname{div} \mathbf{v}) & =-\langle g, \mathbf{n} \cdot \mathbf{v}\rangle & & \forall \mathbf{v} \in H(\operatorname{div}, \Omega) \\
(\operatorname{div} \mathbf{u}, q) & =(f, q) & & \forall q \in L^{2}(\Omega) .
\end{aligned}
$$

Dirichlet boundary conditions are expressed in the right-hand side of (2.2a) throughout the boundary functional

$$
\langle g, \mathbf{n} \cdot \mathbf{v}\rangle=\sum_{e \subset \Gamma} \int_{e} g \mathbf{n}_{e} \cdot \mathbf{v} \mathrm{d} S,
$$

where $\mathbf{n}_{e}$ represents the unit normal to the boundary face $e \subset \Gamma$ pointing out of the polygonal domain $\Omega$.

Both problems (2.1a) $-(2.1 \mathrm{c}),(2.2 \mathrm{a})$ and $(2.2 \mathrm{~b})$ with (2.3) are well-posed and admits a unique solution under the usual assumptions: $f \in L^{2}(\Omega), g \in H^{\frac{1}{2}}(\Gamma)$, K strongly elliptic with the components in $L^{\infty}(\Omega)$. We recall that the tensor field $\mathrm{K}$ is strongly elliptic if there are two positive constants $\kappa_{*}$ and $\kappa^{*}$ such that:

$$
\kappa_{*}\|\mathbf{v}\|^{2} \leq \mathbf{v}^{T} \mathrm{~K} \mathbf{v} \leq \kappa^{*}\|\mathbf{v}\|^{2} \quad \forall \mathbf{v} \in \mathbb{R}^{d} .
$$

For the convergence analysis of Sections 4 and 5, we need the stronger regularity assumption that for every mesh cell the components of $\mathrm{K}$ is locally in $W^{k+2, \infty}$.

To formulate the mixed mimetic approximation of problems (2.2a) and (2.2b), we need to introduce a few mathematical objects, whose precise definition will be presented in the rest of this section. On a sequence of mesh partitions of $\Omega$ satisfying some regularity assumptions and characterized by the mesh size parameter $h$, 
we consider the discrete analogs $p_{h}, q_{h}$ and $f_{h}$ of the scalar functions $p, q$ and $f$, and $\mathbf{u}_{h}, \mathbf{v}_{h}$ of the vector functions $\mathbf{u}, \mathbf{v}$. We assume that $p_{h}$ and $q_{h}$ belong to the linear space $Q_{h}$, which is referred to as the space of the scalar grid functions, and that $\mathbf{u}_{h}$ and $\mathbf{v}_{h}$ belong to the linear space $X_{h}$, which is referred to as the space of the flux grid functions. The grid functions in $Q_{h}$ and $X_{h}$ are vectors of numbers, the degrees of freedom, and will be used to approximate pressure and flux, respectively. We equip $X_{h}$ and $Q_{h}$ with the mimetic inner products $[\cdot, \cdot]_{X_{h}}$ and $[\cdot, \cdot]_{Q_{h}}$. The discrete mimetic gradient operator $\mathcal{G} \mathcal{R} \mathcal{A D}: Q_{h} \rightarrow X_{h}$ can be introduced to approximate the gradient operator $\nabla$ and defined as $\mathcal{G} \mathcal{R} \mathcal{A D}=-\mathcal{D I} \mathcal{V}^{*}$, i.e., as the adjoint $\mathcal{D} \mathcal{I} \mathcal{V}^{*}$ of the discrete divergence operator $\mathcal{D I V}: X_{h} \rightarrow Q_{h}$ that approximates the divergence operator div. As it was show in [19], the discrete gradient operator leads to an equivalent weak formulation that has a saddle-point structure. For this reason, we do not introduce the discrete gradient operator here, but we consider the formulation with the discrete divergence operator.

Now, the mixed MFD method reads as:

$$
\begin{aligned}
& \text { find }\left(\mathbf{u}_{h}, p_{h}\right) \in X_{h} \times Q_{h} \text { such that } \\
& \qquad \begin{array}{rr}
{\left[\mathbf{u}_{h}, \mathbf{v}_{h}\right]_{X_{h}}-\left[p_{h}, \mathcal{D} \mathcal{I} \mathcal{V} \mathbf{v}_{h}\right]_{Q_{h}}=-\left\langle g, \mathbf{v}_{h}\right\rangle_{h}} \\
{\left[\mathcal{D} \mathcal{I} \mathcal{V} \mathbf{u}_{h}, q_{h}\right]_{Q_{h}}=\left[f_{h}, q_{h}\right]_{Q_{h}}}
\end{array} \\
& \quad \forall \mathbf{v}_{h} \in X_{h},
\end{aligned}
$$

The Dirichlet boundary condition (2.1c) is expressed in the right-hand side of (2.5a) by

$$
\left\langle g, \mathbf{v}_{h}\right\rangle_{h}=\sum_{e \subset \Gamma} \int_{e} g \widetilde{v}_{h}^{e} \mathrm{~d} S,
$$

where the boundary function $\widetilde{v}_{h}^{e}$ is the polynomial interpolation in $\mathbb{P}_{k+1}(e)$ built using the degrees of freedom of $\mathbf{v}_{h}$ on $e$.

\subsection{Notation and basic assumptions}

Let $\Omega_{h}$ be a partition of the domain $\Omega$ consisting of polygons for $d=2$ and polyhedra for $d=3$. We denote by $\mathcal{E}^{h}$ the set of all the edges/faces in $\Omega_{h}$, and let $\mathcal{E}_{0}^{h}=\mathcal{E}^{h} \backslash \partial \Omega$ the set of all interior edges/faces. For every polygon/polyhedron $E \in \Omega_{h}$, we denote by $|E|$ its Lebesgue measure (area/volume), by $\partial E$ its boundary, and by $h_{E}$ its diameter. Similarly, for every edge/face $e \in \mathcal{E}^{h}$, we denote by $|e|$ the measure (lenght/area) of $e$, by $h_{e}$ its characteristic lenght, and by $\mathbf{n}_{e}$ its unit normal vector, whose orientation is assumed once and for all. When $e$ is considered on the boundary of cell $E$, its normal vector is denoted by $\mathbf{n}_{E, e}$ and is always pointing out of $E$. When $d=2$, we take $h_{e}=|e|$, while, for $d=3$, we take $h_{e}$ equal to the diameter of the polygonal face $e$. We use $\xi$ and $(\xi, \eta)$ to denote local Cartesian coordinates defined on $e$ for $d=2$ and 3, respectively. We also set as usual the mesh size of $\Omega_{h}$ by

$$
h=\max _{E \in \Omega_{h}} h_{E} .
$$

We assume that all the elements of $\Omega_{h}$ are closed and simply connected subset of $\mathbb{R}^{d}$, all polyhedral faces are flat and all mesh edges are straight segments.

The analysis of the mimetic finite difference method requires some regularity assumptions for the sequence of partitions $\left\{\Omega_{h}\right\}_{h}$ when $h \rightarrow 0$, which we list below only for $d=3$ (for $d=2$ they are readily obtained by reduction).

- (HG) Star-shape regularity: there exist a positive integer number $N_{s}$ and a positive real number $\rho_{s}>0$ such that every mesh $\Omega_{h}$ admits a sub-partition $\mathcal{S}^{h}$ into shape-regular tetrahedra such that:

- (HG1) every polyhedron $E \in \Omega_{h}$ has Lipschitz boundary $\partial E$ and admits a decomposition $\mathcal{S}_{E}^{h}$ made of less than $N_{s}$ tetrahedra. 
- (HG2) the shape regularity of the tetrahedra $E \in \mathcal{S}_{E}^{h}$ is defined as follows: the ratio between the radius $r_{\mathrm{T}}$ of the inscribed ball and the diameter $h_{\mathrm{T}}$ of the tetrahedron $\mathrm{T}$ is bounded from below by $\rho_{s}$.

- (HG3) There exists a positive number $\tau^{*}$ such that each element is star-shaped with respect to all points of a ball of radius $\tau^{*} h_{E}$ centered at an internal point of $E$.

From the above assumptions several properties of the mesh, which are useful in the analysis of the MFD scheme, can be derived. We list them below for the sake of the reader's convenience and for future reference in the paper; proofs can be found in [11].

- (M1) There exist two integer numbers $N_{\mathcal{F}}$ and $N_{\mathcal{E}}$ such that every element $E$ has at most $N_{\mathcal{F}}$ faces and every face has at most $N_{\mathcal{E}}$ edges.

- (M2) There exists a constant $a^{*}$ independent of $h_{E}$ and the mesh $\Omega_{h}$ such that

$$
a^{*} h_{E}^{d-1} \leq|e|, \quad a^{*} h_{E} \leq h_{e}, \quad a^{*} h_{E} \leq|l|,
$$

where $l \in \partial E$ is an edge of the polygonal boundary of face $e$. Roughly speaking, we state that for every element the area of each element's face and the lenght of each element's edge scale properly with respect to the element's diameter.

- (M3) Agmon inequality: there exists a constant $C^{A g m}$ independent of $h_{E}$ and the mesh $\Omega_{h}$ such that

$$
\sum_{e \in \partial E}\|\phi\|_{0, e}^{2} \leq C^{A g m}\left(h_{E}^{-1}\|\phi\|_{0, E}^{2}+h_{E}|\phi|_{1, E}^{2}\right)
$$

for any function $\phi \in H^{1}(E)$.

- (M4) Local interpolation: let $m \geq 0$ be an integer number. For any function $q \in H^{m+3}(E)$ there exists a local polynomial approximation of degree $m+2$ defined on $E$, which we denote by $\widehat{q}$. The following estimate of the interpolation error also holds

$$
\|q-\widehat{q}\|_{0, E}+\sum_{l=1}^{m+2} h_{E}^{l}|q-\widehat{q}|_{l, E} \leq C^{I n t p} h_{E}^{k+3}|q|_{m+3, E}
$$

where $C^{I n t p}>0$ is a constant independent of $h_{E}$.

Remark 2.1. The constants $N_{\mathcal{F}}, N_{\mathcal{E}}, a^{*}, C^{A g m}$ and $C^{\text {Intp }}$, which appear in (M1)-(M4) above, may only depend on the constants $N_{s}$ and $\rho_{s}$ of (HG) and the shape of the domain $\Omega$. Assumption (HG3) is used in the analysis section, e.g. in Lemma 3.1.

Throughout the paper, we will make use of the standard notation for Sobolev spaces; hence, if $l>0$ is an integer number and $D$ is $\Omega$ or a cell $E$, then $H^{l}(D)$ denotes the space of square integrable functions with square integrable derivatives up to order $l$ defined on $D$.

The usual definition also holds for $L^{2}(D),\|\cdot\|_{0, D},|\cdot|_{0, D}$, and $H(\operatorname{div}, D)$. We will also use the functional space of vector fields:

$$
V_{\Omega_{h}}=\left\{\mathbf{v} \in H(\operatorname{div}, \Omega) \text { such that } \mathbf{v}_{\left.\right|_{E}} \in H(\operatorname{div}, E) \cap\left(L^{s}(E)\right)^{d} \text { with } s>2 \text { for every } E \in \Omega_{h}\right\},
$$

where $\mathbf{v}_{\left.\right|_{E}}$ is the restriction of $\mathbf{v}$ to $E$. The regularity that is required to the vector fields in $V_{\Omega_{h}}$ is stronger than just being in $H(\operatorname{div}, \Omega)$ and is needed to define the interpolation of $\mathbf{n}_{e} \cdot \mathbf{v}$ on a single edge/face $e \in \mathcal{E}^{h}$. An explanation can be found in [15]. 


\subsection{Polynomial spaces and orthogonal basis functions}

We will find it convenient to consider two different kinds of polynomial spaces, respectively defined on the mesh cells and the mesh edges/faces.

For the mesh cell $E$ and the non-negative integer number $l, \mathbb{P}_{l}(E)$ is the space of the polynomial functions defined on $E$ with degree at most $l$. The dimension of $\mathbb{P}_{l}(E)$ is equal to $n_{l}^{E}=(l+1)(l+2) / 2$ for $d=2$ and $n_{l}^{E}=(l+1)(l+2)(l+3) / 6$ for $d=3$. The set of $n_{l}^{E}$ basis functions $\left\{\varphi_{E, i}\right\}_{i=0, \ldots, n_{l}^{E}-1}$ that satisfies the orthogonality condition

$$
\int_{E} \varphi_{E, i} \varphi_{E, j} \mathrm{~d} V=|E| \delta_{i j}
$$

generates $\mathbb{P}_{l}(E)$. We assume that $\varphi_{E, 0}=1$. The polynomial functions $\varphi_{E, i}$ are practically built by the GramSchmidt orthogonalization process applied to the monomials $1, x, y, x^{2}$, etc., after translation to the barycenter $\mathbf{x}_{E}$ and rescaling by $h_{E}$.

We define the projection $\Pi_{l}^{E}(\phi) \in \mathbb{P}_{l}(E)$ of a scalar function $\phi \in L^{2}(E)$ by the orthogonality relation

$$
\int_{E}\left(\Pi_{l}^{E}(\phi)-\phi\right) \varphi \mathrm{d} V=0 \quad \text { for every } \varphi \in \mathbb{P}_{l}(E),
$$

and the projection $\Pi_{l}^{E}(\phi) \in\left(\mathbb{P}_{l}(E)\right)^{d}$ of the vector function $\phi \in\left(L^{2}(E)\right)^{d}$ by the orthogonality relation

$$
\int_{E}\left(\Pi_{l}^{E}(\phi)-\phi\right) \cdot \varphi \mathrm{d} V=0 \quad \text { for every } \varphi \in\left(\mathbb{P}_{l}(E)\right)^{d}
$$

(for simplicity of notation, we use the same symbol " $\Pi_{l}^{E}$ ").

Likewise, for the mesh edge/face $e$ and the non-negative integer number $l, \mathbb{P}_{l}(e)$ is the space of the polynomial functions defined on $e$ with degree at most $l$. The dimension of $\mathbb{P}_{l}(e)$ is equal to $n_{l}^{e}=l+1$ for $d=2$ and $n_{l}^{e}=(l+1)(l+2) / 2$ for $d=3$. The set of $n_{l}^{e}$ functions $\left\{\varphi_{e, i}\right\}_{i=0, \ldots, n_{l}^{e}-1}$ that satisfies the orthogonality condition

$$
\int_{e} \varphi_{e, i} \varphi_{e, j} \mathrm{~d} S=|e| \delta_{i j}
$$

generates $\mathbb{P}_{l}(e)$. For $d=2$, the polynomial functions $\varphi_{e, i}$ are practically built by translating and rescaling the Legendre polynomials, which are normally defined on the interval $[-1,1]$, over the edge $e$. For $d=3$, on each two-dimensional face we apply the Gram-Schmidt orthogonalization process to the monomials $1, \xi, \eta, \xi^{2}$, etc., where $(\xi, \eta)$ are the local coordinates of the face.

We define the projection $\Pi_{l}^{e}(\phi) \in \mathbb{P}_{l}(e)$ of the scalar function $\phi \in L^{2}(e)$ by the orthogonality relation

$$
\int_{e}\left(\Pi_{l}^{e}(\phi)-\phi\right) \varphi \mathrm{d} S=0 \quad \text { for every } \varphi \in \mathbb{P}_{l}(e)
$$

Remark 2.2. Using orthogonal basis functions to generate $\mathbb{P}_{l}(E),\left(\mathbb{P}_{l}(E)\right)^{d}$, and $\mathbb{P}_{l}(e)$ significantly simplifies the implementation and calculation of the projection operators $\Pi_{l}^{E}$ and $\Pi_{l}^{e}$.

\subsection{Scheme formulation}

\subsubsection{Degrees of freedom}

Hereafter, we denote the degree of the polynomials that are used in each cell to construct $Q_{h}$ and $X_{h}$ by $k$ and on each mesh edge/face by $k+1$, where $k$ is a non-negative integer number. Scalar variables and fluxes are represented in the discrete setting by the elements of $Q_{h}$ and $X_{h}$, respectively, which are linear spaces with the usual rules of addition and multiplication by a scalar number. The degrees of freedom are associated with the edges/faces and the interior of the cells as follows. The meaning of these degrees of freedom will become clear in the next subsection, when we define the interpolation operators. 

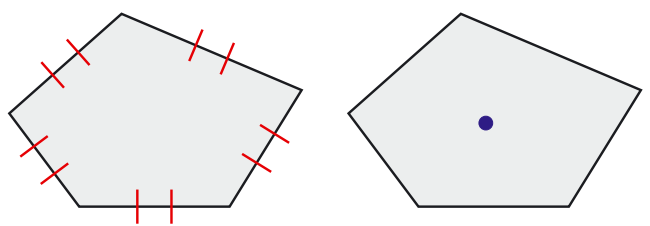

$\mathrm{k}=0$
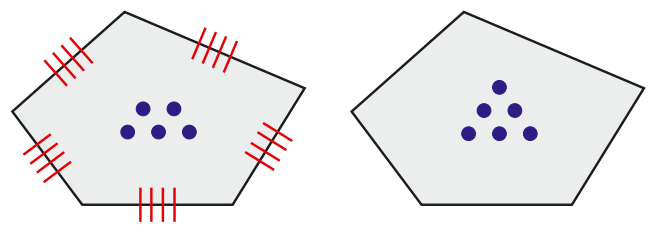

$\mathrm{k}=\mathbf{2}$
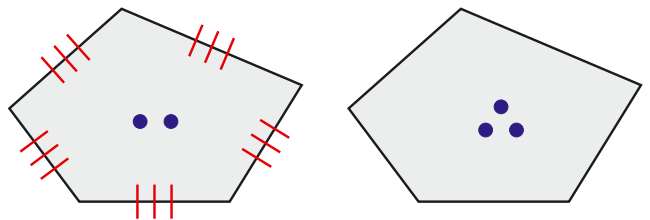

$\mathrm{k}=1$
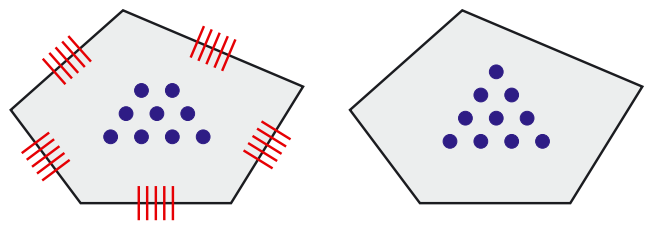

$\mathrm{k}=3$

FiguRE 1. Degrees of freedom for $0 \leq k \leq 3$ on a polygonal cell; for each polynomial degree $k$ we show the flux degrees of freedom on the left and the scalar degrees of freedom on the right. The edge/face moments of the normal component of the flux are denoted by a vertical line; the cell moments are denoted by a bullet.

- Scalar variables. We associate the degrees of freedom of the scalar variable to the mesh cells so that

$$
q_{h} \in Q_{h} \text { means that } q_{h}=\left\{\left\{q_{E, 0}, \cdots q_{E, n_{k}^{E}-1}\right\}_{E \in \Omega_{h}}\right\}
$$

with $q_{E, i} \in \mathbb{R}$. The dimension of $Q_{h}$ is equal to $n_{k}^{E} \times$ number of cells. The restriction of the grid function $q_{h}$ to cell $E$ is given by the set of $n_{k}^{E}$ real numbers $\left\{q_{E, 0}, \cdots q_{E, n_{k}^{E}-1}\right\}$ and denoted by $q_{E}$. The restriction operator that gives the " $E, i$ " th degree of freedom of $q_{h}$ is denoted by $(\cdot)_{E, i}$, i.e., $q_{E, i}=\left(q_{h}\right)_{E, i}$.

- Fluxes. We associate the degrees of freedom of the flux field to the mesh edges/faces and the mesh cells so that

$$
\mathbf{v}_{h} \in X_{h} \text { means that } \mathbf{v}_{h}=\left\{\left\{v_{e, 0}, \ldots v_{e, n_{k+1}^{e}}-1\right\}_{e \in \mathcal{E}_{h}},\left\{v_{E, 1}, \ldots, v_{E, n_{k}^{E}-1}\right\}_{E \in \Omega_{h}}\right\}
$$

with $v_{e, i}, v_{E, i} \in \mathbb{R}$. The dimension of $X_{h}$ is given by $n_{k+1}^{e} \times$ number of edges (2D) or faces (3D) $+\left(n_{k}^{E}-\right.$ $1) \times$ number of cells. The edge/face degrees of freedom of the flux grid function $\mathbf{v}_{h}$ related to $e$ form the set of real numbers $\left\{v_{e, 0}, \ldots v_{e, n_{k+1}^{e}-1}\right\}$, which we denote by $v_{e}$. Similarly, cell degrees of freedom of $\mathbf{v}_{h}$ related to the cell $E$ form the set of real numbers $\left\{v_{E, 1}, \ldots, v_{E, n_{k}^{E}-1}\right\}$, which we denote by $v_{E}$. The restriction of $\mathbf{v}_{h}$ to the mesh cell $E$, denoted by $\mathbf{v}_{E}$, consists of both cell and edge/face degrees of freedom, i.e., $\mathbf{v}_{E}=\mathbf{v}_{\left.h\right|_{E}}=$ $\left(\left\{v_{e}\right\}_{e \in \partial E}, v_{E}\right)$. The restriction operators that give the " $E, i$ " th and " $e, i$ " th degrees of freedom of the vector grid function $\mathbf{v}_{h}$ are respectively denoted by $(\cdot)_{E, i}$ and $(\cdot)_{e, i}, i . e ., v_{E, i}=\left(\mathbf{v}_{h}\right)_{E, i}$ and $v_{e, i}=\left(\mathbf{v}_{h}\right)_{e, i}$.

\subsubsection{Interpolation operators}

The local interpolant of the scalar function $q \in L^{2}(E)$ is the grid function $q_{E}^{\mathrm{I}} \in Q_{E}$ whose components are the moments of $q$ with respect to the orthogonal polynomials $\varphi_{E, i}$ of degree up to $k$ :

$$
q_{E, i}^{\mathrm{I}}:=\left(q^{\mathrm{I}}\right)_{E, i}=\frac{1}{|E|} \int_{E} q \varphi_{E, i} \mathrm{~d} V \quad \text { for } i=0, \ldots, n_{k}^{E}-1, \forall E \in \Omega_{h} .
$$

The global interpolant of $q$ is the grid function $q^{\mathrm{I}}$ of $Q_{h}$ whose restriction to each cell $E$ coincides with the local interpolant $q_{E}^{\mathrm{I}}$. 
The local interpolant of the vector field $\mathbf{v} \in H(\operatorname{div}, E) \cap\left(L^{s}(E)\right)^{d}$ with $s>2$ is the grid function $\mathbf{v}_{E}^{\mathrm{I}} \in X_{E}$ whose components are

- the moments of $\mathbf{v}$ with respect to $\nabla \varphi_{E, i}$, the gradients of the orthogonal polynomials forming a basis of $\mathbb{P}_{k}(E)$ :

$$
v_{E, i}^{\mathrm{I}}:=\left(\mathbf{v}^{\mathrm{I}}\right)_{E, i}=\frac{1}{|E|} \int_{E} \mathbf{v} \cdot \nabla \varphi_{E, i} \mathrm{~d} V, \quad \text { for } i=1, \ldots, n_{k}^{E}-1, \forall E \in \Omega_{h} ;
$$

- the moments of $\mathbf{n}_{e} \cdot \mathbf{v}$ for each edge/face $e \in \partial E$ with respect to the orthogonal polynomials $\left\{\varphi_{e, i}\right\}$ forming a basis of $\mathbb{P}_{k+1}(e)$ :

$$
v_{e, i}^{\mathrm{I}}:=\left(\mathbf{v}^{\mathrm{I}}\right)_{e, i}=\frac{1}{|e|} \int_{e} \mathbf{n}_{e} \cdot \mathbf{v} \varphi_{e, i} \mathrm{~d} S, \quad \text { for } i=0, \ldots, n_{k+1}^{e}-1, \forall e \in \mathcal{E}_{h} .
$$

The moments considered in (2.12) and (2.13) provide as many independent conditions on $\mathbf{v}$ as the dimension of $X_{h \mid} E$, the restriction of $X_{h}$ to cell $E$. The global interpolant of $\mathbf{v} \in V_{\Omega_{h}}$ is the grid function $\mathbf{v}^{\mathrm{I}}$ of $X_{h}$ whose restriction to each cell $E$ coincides with the local interpolant $\mathbf{v}_{E}^{\mathrm{I}}$ (we recall that $V_{\Omega_{h}}$ is defined in (2.8)).

Remark 2.3. The degrees of freedom associated with edge/face $e$ are the discrete representation of the normal flux associated with that edge/face. For each internal edge/face $e \subseteq \partial E^{\prime} \cap \partial E^{\prime \prime}$, i.e., shared by the polygons $E^{\prime}$ and $E^{\prime \prime}$, the degrees of freedom are the same for both adjacent polygons and the continuity of the normal flux across $e$ is automatically satisfied. This construction is consistent with the continuity of the normal component $\mathbf{n}_{e} \cdot \mathbf{v}$ of a vector function $\mathbf{v} \in H(\operatorname{div}, \Omega)$.

Remark 2.4. The definition of the interpolation operator for the scalar variable suggests the following piecewise polynomial representation using the degrees of freedom of the grid function $q_{h}$ and the orthogonal polynomials $\varphi_{E, i}$ inside each cell $E \in \Omega_{h}$ :

$$
\widetilde{q}_{h}(\mathbf{x})=\sum_{i=0}^{n_{k}^{E}-1} q_{E, i} \varphi_{E, i}(\mathbf{x}) \quad \text { for } \mathbf{x} \in E .
$$

Obviously, it holds that $q_{h}=\left(\widetilde{q}_{h}\right)^{\mathrm{I}}$. When $q_{h}=q^{\mathrm{I}}$, the restriction of $\widetilde{q}_{h}$ to $E$ is the $L^{2}$ orthogonal projection of $q$ onto $\mathbb{P}_{k}(E)$, i.e., $\widetilde{\left(q_{E}^{\mathrm{I}}\right)}=\Pi_{k}^{E}(q)$. Hereafter, the symbol "tilde", e.g., ? indicates a polynomials that is built by linear combination of the orthogonal basis functions using the degrees of freedom as coefficients.

Remark 2.5. The definition of the interpolation operator for the flux variable suggests the following piecewise polynomial representation on each edge/face $e$ using the corresponding degrees of freedom of $\mathbf{v}_{h}$ and the orthogonal polynomials $\varphi_{e, i}$

$$
\widetilde{v}_{h}^{e}(\xi)=\sum_{i=0}^{n_{k+1}^{e}-1} v_{e, i} \varphi_{e, i}(\xi) \quad \text { for } \xi \in e
$$

When $\mathbf{v}_{h}=\mathbf{v}^{\mathrm{I}}$, the polynomial $\widetilde{v}_{h}^{e}$ is the $L^{2}$ orthogonal projection of $\mathbf{n}_{e} \cdot \mathbf{v}$ onto $\mathbb{P}_{k+1}(e)$, i.e., $\left(\widetilde{\left.\mathbf{n}_{E, e} \cdot \mathbf{v}\right)^{\mathrm{I}}}=\right.$ $\Pi_{k+1}^{e}\left(\mathbf{n}_{E, e} \cdot \mathbf{v}\right)$.

\subsubsection{Discrete divergence operator}

The discrete divergence operator $\mathcal{D I V}: X_{h} \rightarrow Q_{h}$ is defined cell-wise from the commutation property:

$$
\mathcal{D} \mathcal{I} \mathcal{V} \mathbf{v}^{\mathrm{I}}=(\operatorname{div} \mathbf{v})^{\mathrm{I}} \quad \text { for every } \mathbf{v} \in V_{\Omega_{h}} .
$$


The right-hand side of (2.15) is computable on each cell $E$ by using only the degrees of freedom of $\mathbf{v}_{E}^{\mathrm{I}}$. Indeed, an integration by parts yields:

$$
(\operatorname{div} \mathbf{v})_{E, i}^{\mathrm{I}}=\frac{1}{|E|} \int_{E}(\operatorname{div} \mathbf{v}) \varphi_{E, i} \mathrm{~d} V=\frac{1}{|E|}\left(-\int_{E} \mathbf{v} \cdot \nabla \varphi_{E, i} \mathrm{~d} V+\sum_{e \in \partial E} \int_{e} \mathbf{n}_{E, e} \cdot \mathbf{v} \varphi_{E, i} \mathrm{~d} S\right),
$$

for $i=0, \ldots, n_{k}^{E}-1$. Moreover, taking $i=0$ in the development above shows that our definition is consistent with the Gauss divergence theorem. Since $\left(\mathcal{D} \mathcal{I} \mathcal{V} \mathbf{v}_{h}\right)_{E}$, the restriction of $\mathcal{D} \mathcal{I} \mathcal{V} \mathbf{v}_{h}$ to $E$, belongs to $Q_{E}$, we can consider the polynomial representation in $\mathbb{P}_{k}(E)$ given by

$$
\widetilde{\mathcal{D I} \mathcal{V} \mathbf{v}_{h}}(\mathbf{x})=\sum_{i=0}^{n_{k}^{E}-1}\left(\mathcal{D} \mathcal{I} \mathcal{V} \mathbf{v}_{h}\right)_{E, i} \varphi_{E, i}(\mathbf{x}) \quad \text { for } \mathbf{x} \in E
$$

and, clearly, when $\mathbf{v}_{h}=\mathbf{v}^{\mathrm{I}}$ it holds that $\widetilde{\mathcal{D} \widetilde{\mathcal{I} V \mathbf{v}^{\mathrm{I}}}}{ }_{E}=\Pi_{k}^{E}(\operatorname{div} \mathbf{v})$.

Finally, suppose that $\mathbf{u}_{h}$ is the mimetic flux solving (2.5a) and (2.5b) and $\mathbf{u}^{\mathrm{I}}$ is the interpolant of the flux $\mathbf{u}$ solving problems (2.1a)-(2.1c). For every $q_{h} \in Q_{h}$ it holds that

$$
\left[\mathcal{D I V} \mathbf{u}_{h}, q_{h}\right]_{Q_{h}}=\left[f^{\mathrm{I}}, q_{h}\right]_{Q_{h}}=\left[(\operatorname{div} \mathbf{u})^{\mathrm{I}}, q_{h}\right]_{Q_{h}}=\left[\mathcal{D} \mathcal{I} \mathcal{V} \mathbf{u}^{\mathrm{I}}, q_{h}\right]_{Q_{h}},
$$

from which we obtain the orthogonality property:

$$
\left[\mathcal{D I} \mathcal{V}\left(\mathbf{u}_{h}-\mathbf{u}^{\mathrm{I}}\right), q_{h}\right]_{Q_{h}}=0 \quad \text { for every } q_{h} \in Q_{h}
$$

This property is used in the error analysis of Section 4.

\subsubsection{Mimetic inner product for scalar grid functions}

We define the mimetic inner product in $Q_{h}$ by assembling the mimetic inner products that are locally defined on every cell $E$ :

$$
\left[p_{h}, q_{h}\right]_{Q_{h}}=\sum_{E \in \Omega_{h}}\left[p_{h}, q_{h}\right]_{E}=\sum_{E \in \Omega_{h}}|E| \sum_{i=0}^{n_{k}^{E}-1} p_{E, i} q_{E, i} \text { for every } p_{h}, q_{h} \in Q_{h} .
$$

We also denote the mesh-dependent norm induced by the local mimetic inner product in $Q_{E}$ by $\mid\|\cdot\| \|_{E}$ and the mesh-dependent norms induced by the global inner product in $Q_{h}$ by $\|\cdot\| \|_{Q_{h}}$.

Relation (2.17) corresponds to the $L^{2}$-scalar product for piecewise polynomial functions of degree $k$ associated with $p_{h}$ and $q_{h}$. Indeed,

$$
\int_{E} \widetilde{q}_{h} \widetilde{p}_{h} \mathrm{~d} V=\sum_{i, j=0}^{n_{k}^{E}-1} q_{E, i} p_{E, i} \int_{E} \varphi_{E, i} \varphi_{E, j} \mathrm{~d} V=\sum_{i, j=0}^{n_{k}^{E}-1} q_{E, i} p_{E, i}|E| \delta_{i j}=|E| \sum_{i=0}^{n_{k}^{E}-1} p_{E, i} q_{E, i} .
$$

Consequently, for any pair of square integrable functions $q$ and $p$, the mimetic inner product of their local interpolants $q_{E}^{\mathrm{I}}$ and $p_{E}^{\mathrm{I}}$ is equal to the $L^{2}$ inner products of their orthogonal projection on the polynomial space $\mathbb{P}_{k}(E)$. Using the global interpolations, we have the formal relation

$$
\left[p^{\mathrm{I}}, q^{\mathrm{I}}\right]_{Q_{h}}=\sum_{E \in \Omega_{h}} \int_{E} \Pi_{k}^{E}(p) \Pi_{k}^{E}(q) \mathrm{d} V \quad \text { for every } p, q \in L^{2}(\Omega) .
$$




\subsubsection{Mimetic inner product for flux grid functions}

We define the mimetic inner product in $X_{h}$ by assembling the element-wise contribution from each mesh element $E$

$$
\left[\mathbf{u}_{h}, \mathbf{v}_{h}\right]_{X_{h}}=\sum_{E \in \Omega_{h}}\left[\mathbf{u}_{h}, \mathbf{v}_{h}\right]_{E} \quad \text { for every } \mathbf{u}_{h}, \mathbf{v}_{h} \in X_{h} .
$$

We also denote the mesh-dependent norm induced by the local mimetic inner product in $X_{E}$ by $\left|\|\cdot \mid\| \|_{E}\right.$ and the mesh-dependent norm induced by the global inner product in $X_{h}$ by $\|\cdot \mid \cdot\|_{X_{h}}$.

Remark 2.6. We abuse the notation by using the same symbols $[\cdot, \cdot]_{E}$ and \|\|$\cdot\|\|_{E}$ to denote the local inner products and norms for scalar and vector grid functions. Note that these operators are contextually determined by the nature of their arguments without any ambiguity.

Moreover, both $[\cdot, \cdot]_{E}$ and $\||\cdot|\|_{E}$ only depends on the degrees of freedom of cell $E$. We will keep this dependence implicit through the more elegant notation $\left[p_{h}, q_{h}\right]_{E}$ and $\left\|q_{h}\right\| \|_{E}$ for scalars and $\left[\mathbf{u}_{h}, \mathbf{v}_{h}\right]_{E}$ and $\left\|\left|\mathbf{v}_{h}\right|\right\|_{E}$ for vectors instead of $\left[\left(p_{h}\right)_{E},\left(q_{h}\right)_{E}\right]_{E},\left[\left(\mathbf{u}_{h}\right)_{E},\left(\mathbf{v}_{h}\right)_{E}\right]_{E}$ or $\left[p_{E}, q_{E}\right]_{E},\left[\mathbf{u}_{E}, \mathbf{v}_{E}\right]_{E}$, and $\left|\left\|\left(q_{h}\right)_{E}\right\|\left\|_{E},\right\|\left\|q_{E} \mid\right\| \|_{E}\right.$ or $\left\|\left|\left(\mathbf{v}_{h}\right)_{E}\right|\right\|_{E},\left\|\left|\mathbf{v}_{E}\right|\right\|_{E}$, which are more precise but also more cumbersome.

Now, we present the construction of the local mimetic inner product for fluxes. Consider the cell $E$ and the functional space of vector fields $S_{\Omega_{h}}$, which is a sub-space of $V_{\Omega_{h}}$ and is defined as

$$
S_{\Omega_{h}}=\left\{\mathbf{v} \in V_{\Omega_{h}} \text { such that }(\operatorname{div} \mathbf{v})_{E} \in \mathbb{P}_{k}(E), \mathbf{n}_{E, e} \cdot \mathbf{v} \in \mathbb{P}_{k+1}(e) \text { for every } E \in \Omega_{h}\right\} .
$$

The local inner product $[\cdot, \cdot]_{E}$ is required to satisfy the two following conditions:

- (S1) stability: there exist two constants $\sigma_{*}, \sigma^{*}>0$ independent of $h$ such that

$$
\sigma_{*}|E|\left|\mathbf{v}_{h}\right|_{E}^{2} \leq\left[\mathbf{v}_{h}, \mathbf{v}_{h}\right]_{E} \leq \sigma^{*}|E|\left|\mathbf{v}_{h}\right|_{E}^{2}
$$

for all $\mathbf{v}_{h} \in X_{E}$, where

$$
\left|\mathbf{v}_{h}\right|_{E}^{2}=h_{E}^{2} \sum_{i=0}^{n_{k}^{E}-1}\left|v_{E, i}\right|^{2}+\sum_{e \in \partial E} \sum_{i=0}^{n_{k+1}^{e}-1}\left|v_{e, i}\right|^{2} .
$$

- (S2) local consistency: for every polynomial $q \in \mathbb{P}_{k+2}(E)$ and every function $\mathbf{v} \in S_{\Omega_{h}}$ it holds:

$$
\left[\left(\Pi_{k+1}^{E}(\mathrm{~K} \nabla q)\right)^{\mathrm{I}}, \mathbf{v}^{\mathrm{I}}\right]_{E}=\int_{E} \nabla q \cdot \mathbf{v} \mathrm{d} V .
$$

Since $\mathbf{v} \in S_{\Omega_{h}}$, we integrate by parts (2.23) on cell $E$ and note that in the right integrals we can substitute $\operatorname{div} \mathbf{v}$ and $\mathbf{v} \cdot \mathbf{n}_{E, e}$ with the projections $\Pi_{k}^{E}(\operatorname{div} \mathbf{v})$ and $\Pi_{k+1}^{E}\left(\mathbf{v} \cdot \mathbf{n}_{E, e}\right)$ and then with $\widetilde{\mathcal{D I V} \mathbf{v}^{\mathrm{I}}}$ and $\left(\widetilde{v^{\mathrm{I}}}\right)^{e}$ :

$$
\begin{aligned}
{\left[\left(\Pi_{k+1}^{E}(\mathrm{~K} \nabla q)\right)^{\mathrm{I}}, \mathbf{v}^{\mathrm{I}}\right]_{E} } & =-\int_{E} q \operatorname{div} \mathbf{v} \mathrm{d} V+\sum_{e \in \partial E} \int_{e} \mathbf{v} \cdot \mathbf{n}_{E, e} q \mathrm{~d} S \\
& =-\int_{E} q \Pi_{k}^{E}(\operatorname{div} \mathbf{v}) \mathrm{d} V+\sum_{e \in \partial E} \int_{e} \Pi_{k+1}^{e}\left(\mathbf{v} \cdot \mathbf{n}_{E, e}\right) q \mathrm{~d} S \\
& =-\int_{E} q \widetilde{\mathcal{D I V} \mathbf{v}^{\mathrm{I}}} \mathrm{d} V+\sum_{e \in \partial E} \int_{e}\left(\widetilde{v^{\mathrm{I}}}\right)^{e} q \mathrm{~d} S .
\end{aligned}
$$

Thus, for any grid function $\mathbf{v}_{h} \in X_{E}$, we consider the formula:

$$
\left[\left(\Pi_{k+1}^{E}(\mathrm{~K} \nabla q)\right)^{\mathrm{I}}, \mathbf{v}_{h}\right]_{E}=-\int_{E} q \widetilde{\mathcal{D I V} \mathbf{v}_{h}} \mathrm{~d} V+\sum_{e \in \partial E} \int_{e} \widetilde{v}_{h}^{e} q \mathrm{~d} S .
$$


Remark 2.7. Local consistency can also be defined by directly assuming that

$$
\left[\left(\Pi_{k+1}^{E}(\mathrm{~K} \nabla q)\right)^{\mathrm{I}}, \mathbf{v}^{\mathrm{I}}\right]_{E}=-\int_{E} q \widetilde{\mathcal{D I V} \mathbf{v}^{\mathrm{I}}} \mathrm{d} V+\sum_{e \in \partial E} \int_{e}\left(\widetilde{v^{\mathrm{I}}}\right)^{e} q \mathrm{~d} S .
$$

for every polynomial $q \in \mathbb{P}_{k+2}(E)$ and every function $\mathbf{v} \in V_{\Omega_{h}}$. This definition is equivalent to (S2) as it leads to the same family of mimetic schemes and is the straightforward generalization of the consistency condition that was considered in $[8,19]$. Condition (S2) emphasizes the fact that the local consistency is an exactness property while (2.25) emphasizes the connection with a discrete integration by parts formula. Moreover, it is worth noting that the definition of the weak gradient in the weak Galerkin method is very similar to the right-hand side of (2.25), $c f$. [52].

The mimetic inner product in $X_{E}$ is given by the $n_{k+1}^{E} \times n_{k+1}^{E}$ symmetric and positive definite matrix $\mathrm{M}_{E}$ :

$$
\left[\mathbf{u}_{h}, \mathbf{v}_{h}\right]_{E}=\left(\mathbf{u}_{E}\right)^{T} \mathrm{M}_{E} \mathbf{v}_{E},
$$

where $\mathbf{u}_{E}$ and $\mathbf{v}_{E}$ are the vectors of degrees of freedom of $\mathbf{u}_{h}$ and $\mathbf{v}_{h}$ pertinent to $E$. The convergence properties of the MFD method only depend on the fact that the local scalar product satisfies assumptions (S1) and (S2), and not on the specific form of matrix $M_{E}$. The construction of matrix $M_{E}$ is carried out through the auxiliary matrices $\mathrm{N}$ and $\mathrm{R}$. The $i$ th columns of $\mathrm{N}$ (for $\left.i=1, \ldots, n_{k+2}^{E}-1\right)$, denoted by $\mathrm{N}_{i}$, is given by:

$$
\mathrm{N}_{i}=\left(\Pi_{k+1}^{E}\left(\mathrm{~K} \nabla \varphi_{E, i}\right)\right)^{\mathrm{I}}
$$

The $i$ th columns of $\mathrm{R}$ (for $i=1, \ldots, n_{k+2}^{E}-1$ ), denoted by $\mathrm{R}_{i}$, is such that

$$
\mathbf{v}_{E}^{T} \mathrm{R}_{i}=-\int_{E} \widetilde{\mathcal{D I V} \mathbf{v}_{h}} \varphi_{E, i} \mathrm{~d} V+\sum_{e \in \partial E} \int_{e} \widetilde{v}_{h}^{e} \varphi_{E, i} \mathrm{~d} S \quad \text { for every } \mathbf{v}_{E} \in X_{E}
$$

Matrix $\mathrm{M}_{E}$ is given by the usual formula for the mimetic inner product matrix:

$$
\mathrm{M}=\mathrm{M}_{0}+\mathrm{M}_{1}=\mathrm{R}\left(\mathrm{N}^{T} \mathrm{R}\right)^{-1} \mathrm{R}^{T}+\mu_{E}\left(\mathrm{I}-\mathrm{N}\left(\mathrm{N}^{T} \mathrm{~N}\right)^{-1} \mathrm{~N}^{T}\right),
$$

where $\mu_{E}$ is a scalar factor, usually the trace of matrix $\mathrm{M}_{0}$. Matrices $\mathrm{M}_{0}$ and $\mathrm{M}_{1}$ ensure the consistency and stability properties of the method stated in (S2) and (S1), respectively. Formula (2.29) provides a convenient choice of the mimetic inner product for the practical implementation in a computer code. Nonetheless, a wider family of mimetic inner products exists that satisfies the stability and consistency conditions above [11].

Remark 2.8. For constant tensors $\mathrm{K}$, we can further simplify $(2.23)$ by removing the projection $\Pi_{k+1}^{E}$ from the left-hand side:

$$
\left[(\mathrm{K} \nabla q)^{\mathrm{I}}, \mathbf{v}^{\mathrm{I}}\right]_{E}=\int_{E} \nabla q \cdot \mathbf{v} \mathrm{d} V
$$

Take $q=\varphi_{E, i}$ and $\mathbf{v}=\nabla \varphi_{E, j}$ in (2.23) or (2.30); as $\mathrm{N}^{T} \mathrm{MN}=\mathrm{N}^{T} \mathrm{R}$ we immediately have the identities

$$
\int_{E} \nabla \varphi_{E, i} \cdot \nabla \varphi_{E, j} \mathrm{~d} V=\left(\mathrm{N}^{T} \mathrm{R}\right)_{i j}=\mathrm{N}_{i}^{T} \mathrm{R}_{j}
$$

Since $i$ and $j$ start from 1 , matrix $\mathrm{N}^{T} \mathrm{R}$ is symmetric and positive definite and, thus, non-singular. Hence, $\left(\mathrm{N}^{T} \mathrm{R}\right)^{-1}$ is well-defined in formula $(2.29)$. 
Remark 2.9. The factor $h_{E}^{2}$ that multiplies each $\left|v_{E, i}\right|^{2}$ in $(2.22)$ is needed to make the two summation terms in the right-hand side to scale consistently with respect to $h_{E}$. This requirement is a consequence of the normalization of the orthogonal polynomials that we use to define the cell and the edge/face degrees of freedom. We could remove this factor by renormalizing $\nabla \varphi_{e, i}$ in the definition of $\mathbf{v}_{e, i}^{\mathrm{I}}$ in (2.13). However, the normalization of the same polynomial basis of $\mathbb{P}_{k}(E)$ would be different in the definition of $Q_{E}$ and $X_{E}$, which might be source of errors and confusion.

Remark 2.10. The mimetic inner product between the two flux grid functions $\mathbf{u}^{\mathrm{I}}$ and $\mathbf{v}^{\mathrm{I}}$ that interpolate the vector fields $\mathbf{u}$ and $\mathbf{v}$ is an approximation of the $\mathrm{K}^{-1}$-weighted $L^{2}$-scalar product between these two vectors for any cell $E \in \Omega_{h}$ :

$$
\left[\mathbf{u}^{\mathrm{I}}, \mathbf{v}^{\mathrm{I}}\right]_{E} \approx \int_{E} \mathrm{~K}^{-1} \mathbf{u} \cdot \mathbf{v} \mathrm{d} V
$$

and, consequently, for the whole domain $\Omega$ :

$$
\left[\mathbf{u}^{\mathrm{I}}, \mathbf{v}^{\mathrm{I}}\right]_{X_{h}} \approx \int_{\Omega} \mathrm{K}^{-1} \mathbf{u} \cdot \mathbf{v} \mathrm{d} V
$$

From the previous discussion, it should be clear that for a constant $\mathrm{K}$ this approximation is exact whenever both vectors are the gradient of a polynomial of degree $k+1$. The information about $K^{-1}$ is embedded inside $[\cdot, \cdot]_{X_{h}}$. In the matrix formula (2.29), $\mathrm{K}^{-1}$ affects matrix $\mathrm{M}_{0}$ through $\left(\mathrm{N}^{T} \mathrm{R}\right)^{-1}$, since $\mathrm{N}$ contains $\mathrm{K}$ in its definition, and matrix $M_{1}$ through the choice of the scaling factor $\mu_{E}$.

\subsection{Well-posedness}

Since the bilinear form $[\cdot, \cdot]_{X_{h}}$ in (2.20) is an inner product, it is coercive on the whole space $X_{h}$, and therefore on the kernel of the discrete divergence operator $\mathcal{D I} \mathcal{V}$. The well-posedness of the mimetic method follows from this fact and the discrete inf-sup condition that will be proved in Section 5.2 (see [15]).

\section{Estimates FOR INTERPOLATION AND PROJECTION OPERATORS}

To ease the notation, we use the symbol " $\lesssim$ " to denote " $\leq C$ ", i.e., "less than or equal to up to the constant $C$ ", where $C$ is strictly positive and independent of the mesh size $h$, variables or other parameters of the inequality. By accurately tracing back the different constants as they appear in the proofs, it is immediate to see that all such constants may depend only on the mesh regularity constants in (M1)-(M4), the strong ellipticity constants $\kappa_{*}$ and $\kappa^{*}$, the norm $\|\mathrm{K}\|_{k+2, \infty, E}$, and the stability constants $\sigma_{*}$ and $\sigma^{*}$.

Lemma 3.1. Let $E$ be a mesh element of $\Omega_{h}$, e a mesh edge/face in $\mathcal{E}^{h}, l$ a non-negative integer and $q$ a function in $H^{l+1}(E)$. Under assumption $(\mathrm{HG})$, we have

$$
\begin{array}{r}
\left\|q-\Pi_{l}^{E}(q)\right\|_{0, E}+h_{E}\left\|\nabla\left(q-\Pi_{l}^{E}(q)\right)\right\|_{0, E} \lesssim h_{E}^{l+1}|q|_{l+1, E} \\
\left\|q-\Pi_{l}^{e}(q)\right\|_{0, e} \lesssim h_{e}^{l+1}|q|_{l+1, e} .
\end{array}
$$

Proof. These estimates are the consequence of the mesh regularity assumptions that make it possible to use standard results from polynomial approximation in Sobolev spaces in star-shaped domains [16].

Lemma 3.2. Let $E$ be a mesh element, e be a mesh edge/face that belongs to $\partial E$, and $q$ be a function in $H^{k+3}(E)$ for $k$ non-negative integer. From assumption $(\mathrm{HG})$ and $\mathrm{K}_{i j} \in W^{k+2, \infty}(E)$, it follows that

$$
\begin{array}{r}
\|q-\widehat{q}\|_{0, E}^{2}+h_{E} \sum_{e \in \partial E}\|q-\widehat{q}\|_{0, e}^{2} \lesssim h_{E}^{2(k+3)}\|q\|_{k+3, E}^{2} \\
\left\|\mathrm{~K} \nabla q-\Pi_{k+1}^{E}(\mathrm{~K} \nabla \widehat{q})\right\|_{0, E}^{2}+h_{E} \sum_{e \in \partial E}\left\|\mathrm{~K} \nabla q-\Pi_{k+1}^{E}(\mathrm{~K} \nabla \widehat{q})\right\|_{0, e}^{2} \lesssim h_{E}^{2(k+2)}\|q\|_{k+3, E}^{2}
\end{array}
$$

where $\widehat{q}$ is the polynomial interpolant of degree $k+2$ of $q$ on $E$ defined in (M4). 
Proof. Inequality (3.1a) follows directly from combining (M3) and (M4).

To prove inequality $(3.1 \mathrm{~b})$ we add and subtract $\mathrm{K} \nabla \widehat{q}$ to the two terms of the left-hand side and use the triangle inequality to obtain:

$$
\left\|\mathrm{K} \nabla q-\Pi_{k+1}^{E}(\mathrm{~K} \nabla \widehat{q})\right\|_{0, E}^{2} \lesssim\|\mathrm{K} \nabla(q-\widehat{q})\|_{0, E}^{2}+\left\|\mathrm{K} \nabla \widehat{q}-\Pi_{k+1}^{E}(\mathrm{~K} \nabla \widehat{q})\right\|_{0, E}^{2}
$$

and, similarly,

$$
\left\|\mathrm{K} \nabla q-\Pi_{k+1}^{E}(\mathrm{~K} \nabla \widehat{q})\right\|_{0, e}^{2} \lesssim\|\mathrm{K} \nabla(q-\widehat{q})\|_{0, e}^{2}+\left\|\mathrm{K} \nabla \widehat{q}-\Pi_{k+1}^{E}(\mathrm{~K} \nabla \widehat{q})\right\|_{0, e}^{2}
$$

The right-hand side of (3.2) is bounded by applying standard estimates from polynomial interpolation theory and noting that $\|\widehat{q}\|_{k+2, E} \lesssim\|q\|_{k+2, E}$ (recall that $\widehat{q}$ is the polynomial interpolant of $q$ of degree $k+2$ ):

$$
\begin{aligned}
\|\mathrm{K} \nabla(q-\widehat{q})\|_{0, E}^{2}+\left\|\mathrm{K} \nabla \widehat{q}-\Pi_{k+1}^{E}(\mathrm{~K} \nabla \widehat{q})\right\|_{0, E}^{2} & \lesssim h_{E}^{2(k+2)}\left(\kappa^{*}\right)^{2}\|q\|_{k+3, E}^{2}+h_{E}^{2(k+2)}\|\mathrm{K} \nabla \widehat{q}\|_{k+2, E}^{2} \\
& \lesssim h_{E}^{2(k+2)} \max \left(\kappa^{*},\|\mathrm{~K}\|_{k+2, \infty, E}\right)^{2}\|q\|_{k+3, E}^{2} .
\end{aligned}
$$

The first term in the right-hand side of (3.3) is bounded by applying the Agmon inequality with $\phi=\mathrm{K} \nabla(q-\widehat{q})$ and then using the estimate for the interpolation error:

$$
\begin{aligned}
\sum_{e \in E}\|\mathrm{~K} \nabla(q-\widehat{q})\|_{0, e}^{2} & \lesssim h_{E}^{-1}\|\nabla(q-\widehat{q})\|_{0, E}^{2}+h_{E}|\nabla(q-\widehat{q})|_{1, E}^{2} \lesssim h_{E}^{-1}\left(h_{E}^{k+2}|q|_{k+3, E}\right)^{2}+h_{E}\left(h_{E}^{k+1}|q|_{k+3, E}\right)^{2} \\
& \lesssim h_{E}^{2 k+3}|q|_{k+3, E}^{2} .
\end{aligned}
$$

The second term in the right-hand side of (3.3) is bounded by applying the Agmon inequality with $\phi=\mathrm{K} \nabla \widehat{q}-$ $\Pi_{k+1}^{E}(\mathrm{~K} \nabla \widehat{q})$, applying the error estimate for $\Pi_{k+1}^{E}$ of the previous lemma, and noting that $\mathrm{K}_{i j} \in W^{k+2, \infty}(E)$ :

$$
\begin{aligned}
\sum_{e \in \partial E}\left\|\mathrm{~K} \nabla \widehat{q}-\Pi_{k+1}^{E}(\mathrm{~K} \nabla \widehat{q})\right\|_{0, e}^{2} & \lesssim h_{E}^{-1}\left\|\mathrm{~K} \nabla \widehat{q}-\Pi_{k+1}^{E}(\mathrm{~K} \nabla \widehat{q})\right\|_{0, E}^{2}+h_{E}\left|\mathrm{~K} \nabla \widehat{q}-\Pi_{k+1}^{E}(\mathrm{~K} \nabla \widehat{q})\right|_{1, E}^{2} \\
& \lesssim h_{E}^{-1}\left(h_{E}^{k+2}|\mathrm{~K} \nabla \widehat{q}|_{k+2, E}\right)^{2}+h_{E}\left(h_{E}^{k+1}|\mathrm{~K} \nabla \widehat{q}|_{k+2, E}\right)^{2} \\
& \lesssim h_{E}^{2 k+3}\|\mathrm{~K}\|_{k+2, \infty, E}^{2}\|q\|_{k+2, E}^{2} \cdot
\end{aligned}
$$

To estimate the norms and seminorms of $\mathrm{K} \nabla \widehat{q}$ we needed the stronger regularity of $\mathrm{K}$, which we indicates explicitly through the factor $\|\mathrm{K}\|_{k+2, \infty, E}$. The quantity $\max \left(\kappa^{*},\|\mathrm{~K}\|_{k+2, \infty, E}\right)$ that would appear in the final inequality is absorbed by the $\lesssim$ notation in the assertion of the lemma.

Lemma 3.3. Let $E$ be a mesh element, $\mathbf{v}$ a function in $\left(H^{1}(E)\right)^{d}$ and $\mathbf{v}^{I}$ its interpolation in $X_{E}$. Then, it holds that

$$
\left\|\left|\mathbf{v}^{I}\right|\right\|_{E}^{2} \lesssim\|\mathbf{v}\|_{0, E}^{2}+h_{E} \sum_{e \in \partial E}\|\mathbf{v}\|_{0, e}^{2} \lesssim\|\mathbf{v}\|_{0, E}^{2}+h_{E}^{2}|\mathbf{v}|_{1, E}^{2}
$$

Proof. In view of (S1), we only need to prove that

$$
|E|\left|\mathbf{v}^{\mathrm{I}}\right|_{E}^{2} \lesssim\|\mathbf{v}\|_{0, E}^{2}+h_{E} \sum_{e \in \partial E}\|\mathbf{v}\|_{0, e}^{2} \lesssim\|\mathbf{v}\|_{0, E}^{2}+h_{E}^{2}|\mathbf{v}|_{1, E}^{2}
$$


where $\left|\mathbf{v}^{\mathrm{I}}\right|_{E}$ is defined in $(2.22)\left(\right.$ take $\left.\mathbf{v}_{h}=\mathbf{v}^{\mathrm{I}}\right)$. First, note that

$$
|E| h_{E}^{2}\left|v_{E, i}^{\mathrm{I}}\right|^{2}=|E| h_{E}^{2}\left|\frac{1}{|E|} \int_{E} \mathbf{v} \cdot \nabla \varphi_{E, i} \mathrm{~d} V\right|^{2} \leq\|\mathbf{v}\|_{0, E}^{2} \frac{h_{E}^{2}}{|E|}\left\|\nabla \varphi_{E, i}\right\|_{0, E}^{2} \lesssim\|\mathbf{v}\|_{0, E}^{2},
$$

since $h_{E}^{2}\left\|\nabla \varphi_{E, i}\right\|_{0, E}^{2} \lesssim|E|$. From the orthogonality of the basis functions $\varphi_{e, i}$ it follows that:

$$
\begin{aligned}
|e| \sum_{i=0}^{n_{k+1}^{e}-1}\left|v_{e, i}^{\mathrm{I}}\right|^{2} & =\sum_{i, j=0}^{n_{k+1}^{e}-1} v_{e, i}^{\mathrm{I}} v_{e, j}^{\mathrm{I}}|e| \delta_{i j}=\sum_{i, j=0}^{n_{k+1}^{e}-1} v_{e, i}^{\mathrm{I}} v_{e, j}^{\mathrm{I}} \int_{e} \varphi_{e, i} \varphi_{e, j} \mathrm{~d} S \\
& =\int_{e}\left(\sum_{i=0}^{n_{k+1}^{e}-1} v_{e, i}^{\mathrm{I}} \varphi_{e, i}\right)\left(\sum_{j=0}^{n_{k+1}^{e}-1} v_{e, j}^{\mathrm{I}} \varphi_{e, j}\right) \mathrm{d} S=\int_{e}\left|\left(\widetilde{v^{\mathrm{I}}}\right)^{e}\right|^{2} \mathrm{~d} S=\left\|\left(\widetilde{v^{\mathrm{I}}}\right)^{e}\right\|_{0, e}^{2}
\end{aligned}
$$

The mesh regularity implies that $|E| \lesssim|e| h_{E}$. Therefore, as $\left(\widetilde{v^{\mathrm{I}}}\right)^{e}$ is the $L^{2}$ projection of $\mathbf{n}_{e} \cdot \mathbf{v}$ in $\mathbb{P}_{k+1}(e)$, we obtain:

$$
|E| \sum_{i=0}^{n_{k+1}^{e}-1}\left|v_{e, i}^{\mathrm{I}}\right|^{2} \lesssim h_{E}\left\|\left(\widetilde{v^{\mathrm{I}}}\right)^{e}\right\|_{0, e}^{2} \lesssim h_{E}\left\|\Pi_{k+1}^{e}\left(\mathbf{n}_{E, e} \cdot \mathbf{v}\right)\right\|_{0, e}^{2} \lesssim h_{E}\left\|\mathbf{n}_{E, e} \cdot \mathbf{v}\right\|_{0, e}^{2} \lesssim h_{E}\|\mathbf{v}\|_{0, e}^{2}
$$

The second inequality in (3.4) is a consequence of Agmon inequality.

Lemma 3.4. Let $E$ be a mesh element, e be a mesh edge/face that belongs to $\partial E$, and $q$ be a function in $H^{k+3}(E)$ for $k$ non-negative integer. From assumption $(\mathrm{HG})$ and $\mathrm{K}_{i j} \in W^{k+2, \infty}(E)$, it follows that

$$
\left\|\left(\mathrm{K} \nabla q-\Pi_{k+1}^{E}(\mathrm{~K} \nabla \widehat{q})\right)^{I}\right\|_{E} \lesssim h_{E}^{k+2}\|q\|_{k+3, E}
$$

where $\widehat{q}$ is the polynomial interpolant of degree $k+2$ of $q$ on $E$ defined in (M4).

Proof. Combine the first inequality of Lemma 3.3 with $\mathbf{v}=\mathrm{K} \nabla q-\Pi_{k+1}^{E}(\mathrm{~K} \nabla \widehat{q})$ and estimate (3.1b):

$$
\begin{aligned}
\|\|\left(\mathrm{K} \nabla q-\Pi_{k+1}^{E}(\mathrm{~K} \nabla \widehat{q})\right)^{\mathrm{I}} \mid \|_{E}^{2} & \lesssim\left\|\mathrm{K} \nabla q-\Pi_{k+1}^{E}(\mathrm{~K} \nabla \widehat{q})\right\|_{0, E}^{2}+h_{E} \sum_{e \in \partial E}\left\|\mathrm{~K} \nabla q-\Pi_{k+1}^{E}(\mathrm{~K} \nabla \widehat{q})\right\|_{0, e}^{2} \\
& \lesssim h_{E}^{2(k+2)}\|q\|_{k+3, E}^{2} .
\end{aligned}
$$

This ends the proof of the lemma.

\section{Convergence of the flux variable}

The result of this section is the following convergence theorem for the approximation of the flux variable.

Theorem 4.1. Let $(\mathbf{u}, p)$ be the exact solution of problem (2.1a)-(2.1c) with $p \in H^{k+3}(\Omega)$, where $k$ is a nonnegative integer number. Let $\left(\mathbf{u}_{h}, p_{h}\right) \in X_{h} \times Q_{h}$ be the solution of (2.5a) and (2.5b) under assumption $(\mathrm{HG})$. Then,

$$
\left\|\left.\left|\mathbf{u}^{I}-\mathbf{u}_{h}\right|\right|_{X_{h}} \lesssim h^{k+2}\right\| p \|_{k+3, \Omega}
$$


Proof. Denote $\varepsilon_{h}=\mathbf{u}^{\mathrm{I}}-\mathbf{u}_{h}$. Using the orthogonality property (2.16) in (2.5a) (with $\mathbf{v}_{h}=\mathbf{u}^{\mathrm{I}}-\mathbf{u}_{h}$ ) yields:

$$
\left[\mathbf{u}_{h}, \varepsilon_{h}\right]_{X_{h}}=-\left\langle g, \varepsilon_{h}\right\rangle_{h}
$$

Let $\widehat{p}$ be the piecewise polynomial interpolant of degree $k+2$ of $p$ defined on $\Omega_{h}$ in accordance with (M4), i.e., $\widehat{p}_{E}=\widehat{p}_{\left.\right|_{E}}$ interpolates the restriction of $p$ to $E$ in $\mathbb{P}_{k+2}(E)$. Starting from the definition of the norm $\left|\|\cdot \mid\|_{X_{h}}\right.$ we have the development:

$$
\begin{aligned}
\left.\left\|\varepsilon_{h}\right\|\right|_{X_{h}} ^{2} & =\left[\mathbf{u}^{\mathrm{I}}, \boldsymbol{\varepsilon}_{h}\right]_{X_{h}}-\left[\mathbf{u}_{h}, \boldsymbol{\varepsilon}_{h}\right]_{X_{h}} & & {[\text { use }(4.2)] } \\
& =\left[\mathbf{u}^{\mathrm{I}}, \boldsymbol{\varepsilon}_{h}\right]_{X_{h}}+\left\langle g, \boldsymbol{\varepsilon}_{h}\right\rangle_{h} & & {\left[\text { use } \mathbf{u}^{\mathrm{I}}=(-\mathrm{K} \nabla p)^{\mathrm{I}}\right] } \\
& =\left[(-\mathrm{K} \nabla p)^{\mathrm{I}}, \boldsymbol{\varepsilon}_{h}\right]_{X_{h}}+\left\langle g, \boldsymbol{\varepsilon}_{h}\right\rangle_{h} & & {[\text { use }(2.20)] } \\
& =\sum_{E \in \Omega_{h}}\left[(-\mathrm{K} \nabla p)^{\mathrm{I}}, \boldsymbol{\varepsilon}_{h}\right]_{E}+\left\langle g, \boldsymbol{\varepsilon}_{h}\right\rangle_{h} & & {\left[\text { add and subtract }\left(\Pi_{k+1}^{E}(\nabla \widehat{p})\right)^{\mathrm{I}}\right] } \\
& =\mathrm{A}_{1}+\mathrm{A}_{2} & &
\end{aligned}
$$

where

$$
\begin{aligned}
& \mathrm{A}_{1}=\sum_{E \in \Omega_{h}}\left[(-\mathrm{K} \nabla p)^{\mathrm{I}}+\left(\Pi_{k+1}^{E}(\mathrm{~K} \nabla \widehat{p})\right)^{\mathrm{I}}, \varepsilon_{h}\right]_{E}, \\
& \mathrm{~A}_{2}=-\sum_{E \in \Omega_{h}}\left[\left(\Pi_{k+1}^{E}(\mathrm{~K} \nabla \widehat{p})\right)^{\mathrm{I}}, \varepsilon_{h}\right]_{E}+\left\langle g, \varepsilon_{h}\right\rangle_{h} .
\end{aligned}
$$

The proof continues by estimating the error terms $A_{1}$ and $A_{2}$ separately.

We first derive an upper bound for $A_{1}$. From the Cauchy-Schwartz inequality and Lemma 3.4 it follows that

$$
\left[(-\mathrm{K} \nabla p)^{\mathrm{I}}+\left(\Pi_{k+1}^{E}(\mathrm{~K} \nabla \widehat{p})\right)^{\mathrm{I}}, \varepsilon_{h}\right]_{E} \leq\|\|(-\mathrm{K} \nabla p)^{\mathrm{I}}+\left(\Pi_{k+1}^{E}(\mathrm{~K} \nabla \widehat{p})\right)^{\mathrm{I}}\|\|_{E}\left\|\varepsilon_{h}\right\|\left\|_{E} \lesssim h_{E}^{k+2}\right\| p\left\|_{k+3, E}\right\| \varepsilon_{h}\|\|_{E} .
$$

Then, we sum over all the mesh cells and use again the Cauchy-Schwarz inequality to estimate $A_{1}$ :

$$
\begin{aligned}
\left|\mathrm{A}_{1}\right| & \lesssim \sum_{E \in \Omega_{h}} h_{E}^{k+2}\|p\|_{k+3, E}\left|\left\|\varepsilon_{h} \mid\right\|_{E} \lesssim h^{k+2}\left(\sum_{E \in \Omega_{h}}\|p\|_{k+3, E}^{2}\right)^{1 / 2}\left(\sum_{E \in \Omega_{h}}\left\|\varepsilon_{h}\right\|_{E}^{2}\right)^{1 / 2}\right. \\
& \lesssim h^{k+2}\|p\|_{k+3, \Omega}\left\|\left|\varepsilon_{h}\right|\right\|_{X_{h}} .
\end{aligned}
$$

To derive an upper bound for term $\mathrm{A}_{2}$, first observe that the consistency condition (S2) and the orthogonality property (2.16) imply that

$$
\left[\left(\Pi_{k+1}^{E}(\mathrm{~K} \nabla \widehat{p})\right)^{\mathrm{I}}, \varepsilon_{h}\right]_{E}=\sum_{e \in \partial E} \int_{e} \widehat{p} \widetilde{\varepsilon}_{h}^{e} \mathrm{~d} S .
$$

Furthermore, the trace of $p \in H^{k+3}(\Omega)$ is continuous at every internal face and $\widetilde{\varepsilon}_{h}^{e}$ takes opposite values at the sides of every internal face so that

$$
\sum_{E \in \Omega_{h}} \sum_{e \in \partial E} \int_{e} p \widetilde{\varepsilon}_{h}^{e} \mathrm{~d} S=\left\langle p, \varepsilon_{h}\right\rangle_{h}
$$


Now, we use (2.20) and identities (4.6) and (4.5) and the Cauchy-Schwarz inequality twice to obtain

$$
\begin{aligned}
{\left[\left(\Pi_{k+1}^{E}(\mathrm{~K} \nabla \widehat{p})\right)^{\mathrm{I}}, \varepsilon_{h}\right]_{X_{h}}-\left\langle p, \varepsilon_{h}\right\rangle_{h} } & =\sum_{E \in \Omega_{h}}\left[\left(\Pi_{k+1}^{E}(\mathrm{~K} \nabla \widehat{p})\right)^{\mathrm{I}}, \varepsilon_{h}\right]_{E}-\sum_{E \in \Omega_{h}} \sum_{e \in \partial E} \int_{e} p \widetilde{\varepsilon}_{h}^{e} \mathrm{~d} S \\
& =\sum_{E \in \Omega_{h}} \sum_{e \in \partial E} \int_{e}(\widehat{p}-p) \widetilde{\varepsilon}_{h}^{e} \mathrm{~d} S \leq \sum_{E \in \Omega_{h}} \sum_{e \in \partial E}\|\widehat{p}-p\|_{0, e}\left\|\varepsilon_{h}\right\|_{0, e} \\
& \leq \sum_{E \in \Omega_{h}}\left(\sum_{e \in \partial E}\|\widehat{p}-p\|_{0, e}^{2}\right)^{1 / 2}\left(\sum_{e \in \partial E}\left\|\widetilde{\varepsilon}_{h}^{e}\right\|_{0, e}^{2}\right)^{1 / 2} .
\end{aligned}
$$

From Remark 2.4 and the orthogonality of $\varphi_{e, i}(\xi)$ it follows that

$$
\begin{aligned}
\left\|\widetilde{\varepsilon}_{h}^{e}\right\|_{0, e}^{2} & =\int_{e}\left|\widetilde{\varepsilon}_{h}^{e}\right|^{2} \mathrm{~d} \xi=\sum_{i, j=0}^{n_{k+1}^{e}-1}\left(\varepsilon_{h}\right)_{e, i}\left(\varepsilon_{h}\right)_{e, j} \int_{e} \varphi_{e, i}(\xi) \varphi_{e, j}(\xi) \mathrm{d} \xi=\sum_{i, j=0}^{n_{k+1}^{e}-1}\left(\varepsilon_{h}\right)_{e, i}\left(\varepsilon_{h}\right)_{e, j}|e| \delta_{i j} \\
& =\sum_{i}^{n_{k+1}^{e}-1}\left|\left(\varepsilon_{h}\right)_{e, i}\right|^{2}|e| .
\end{aligned}
$$

This relation, assumption (S1) and $h_{E} \lesssim|e| /|E|$ from (M2) imply that

$$
\sum_{e \in \partial E}\left\|\widetilde{\varepsilon}_{h}^{e}\right\|_{0, e}^{2}=\sum_{e \in \partial E}|e| \sum_{i=0}^{n_{k+1}^{e}-1}\left|\left(\varepsilon_{h}\right)_{e, i}\right|^{2} \lesssim h_{E}^{-1}\left\|\mid \varepsilon_{h}\right\|_{E}^{2} .
$$

Since $g=p$ on boundary $\Gamma$, inequality (4.7) combined with inequality (3.1a) and the Cauchy-Schwarz inequality yields the estimate:

$$
\begin{aligned}
\left|\mathrm{A}_{2}\right| & =\left|\sum_{E, \in \Omega_{h}}\left[\left(\Pi_{k+1}^{E}(\mathrm{~K} \nabla \widehat{p})\right)^{\mathrm{I}}, \varepsilon_{h}\right]_{E}-\left\langle g, \varepsilon_{h}\right\rangle_{h}\right| \lesssim \sum_{E \in \Omega_{h}} h_{E}^{k+5 / 2}\|p\|_{k+3, E} h_{E}^{-1 / 2}\left\|\varepsilon_{h}\right\| \|_{E} \\
& \lesssim h^{k+2}\left(\sum_{E \in \Omega_{h}}\|p\|_{k+3, E}^{2}\right)^{1 / 2}\left(\sum_{E \in \Omega_{h}}\left\|\varepsilon_{h}\right\|_{E}^{2}\right)^{1 / 2} \lesssim h^{k+2}\|p\|_{k+3, \Omega}\left\|\varepsilon_{h}\right\| \|_{X_{h}} .
\end{aligned}
$$

The assertion of the theorem follows from combining the estimates for $A_{1}$ and $A_{2}$ in (4.4) and (4.8).

\section{Convergence of the scalar variable}

The main result of this section is the following convergence theorem for the approximation of the scalar variable.

Theorem 5.1. Let $k$ be a non-negative integer number and $p \in H^{k+3}(\Omega)$ be the exact solution of problem (2.1a)-(2.1c). Let $p_{h} \in Q_{h}$ be the solution of (2.5a) and (2.5b) under assumption (HG). Then, there holds

$$
\left\|p^{I}-p_{h}||_{Q_{h}} \lesssim h^{k+2}\right\| p \|_{k+3, \Omega}
$$

The proof of this theorem is postponed to Section 5.3 as it requires the lifting operator and the discrete inf-sup condition, which are presented in Sections 5.1 and 5.2 . 


\subsection{Lifting operator}

Consider $E \in \Omega_{h}$. There exists a local lifting operator $R_{E}: X_{E} \rightarrow H(\operatorname{div}, E)$ such that:

(R1) for every $\mathbf{v}_{h} \in X_{h}$ it holds:

$$
\begin{aligned}
\operatorname{div} R_{E}\left(\mathbf{v}_{h}\right) & =\widetilde{\mathcal{D I V} \mathbf{v}_{h}} & & \text { in } E \\
\mathbf{n}_{E, e} \cdot R_{E}\left(\mathbf{v}_{h}\right) & =\widetilde{v}_{h}^{e} & & \text { on every } e \in \partial E
\end{aligned}
$$

(R2) for every vector field whose components have a $(k+1)$-degree polynomial restriction on E, i.e., $\mathbf{v}_{\left.\right|_{E}} \in$ $\left(\mathbb{P}_{k+1}(E)\right)^{d}$, it holds:

$$
R_{E}\left(\left(\mathbf{v}_{\left.\right|_{E}}\right)^{\mathrm{I}}\right)=\mathbf{v}_{\left.\right|_{E}}
$$

(R3) for every $\mathbf{v}_{h} \in X_{h}$, it holds

$$
\rho_{*}|E|\left|\mathbf{v}_{h}\right|_{E}^{2} \leq\left\|R_{E}\left(\mathbf{v}_{h}\right)\right\|_{0, E}^{2} \leq \rho^{*}|E|\left|\mathbf{v}_{h}\right|_{E}^{2}
$$

where $\left|\mathbf{v}_{h}\right|_{E}^{2}$ is defined in (2.22).

Remark 5.2. The locally lifted field $R_{E}\left(\mathbf{v}_{h}\right)$ only depends on the degrees of freedom of $\mathbf{v}_{h}$ of cell $E$. Here, as before, we prefer to use the more elegant notation $R_{E}\left(\mathbf{v}_{h}\right)$ instead of $R_{E}\left(\left(\mathbf{v}_{h}\right)_{E}\right)$ or $R_{E}\left(\mathbf{v}_{E}\right)$.

Remark 5.3. In view of the stability condition (S1), condition (R3) is equivalent to

$$
\frac{\rho_{*}}{\sigma^{*}}\left\|\mid \mathbf{v}_{h}\right\|_{E}^{2} \leq\left\|R_{E}\left(\mathbf{v}_{h}\right)\right\|_{0, E}^{2} \leq \frac{\rho^{*}}{\sigma_{*}}\left\|\mathbf{v}_{h}\right\|_{E}^{2} .
$$

By combining the local lifting operators, we define a global lifting operator $R: X_{h} \rightarrow\left(L^{2}(\Omega)\right)^{d}$, which is such that $R\left(\mathbf{v}_{h}\right)_{\mid E}=R_{E}\left(\mathbf{v}_{h}\right)$. As is usual in the mimetic technology, the lifting operator is a valuable tool in the convergence analysis, but is not needed in any practical implementation of the method. Furthermore, a lifting operator satisfying the conditions listed above is not unique; however, only its existence is needed in the analysis. The existence can be proved as in ([11], Chap. 3) or through direct construction by solving numerically the system of equations (5.2a) and (5.2b), for example, by the $\mathrm{BDM}_{k+1}-\mathrm{P}_{k}$ scheme, on the sub-partition $\mathcal{S}^{h}$ of assumption (HG).

Using the lifting operator we can prove this lemma, which will be useful in the proof of Theorem 5.1.

Lemma 5.4. Let $(\mathbf{u}, p)$ be the exact solution of problem (2.1a)-(2.1c) with $p \in H^{k+3}(\Omega)$, where $k$ is a nonnegative integer number. Let $\left(\mathbf{u}_{h}, p_{h}\right) \in X_{h} \times Q_{h}$ be the solution of (2.5a) and (2.5b) under assumption $(\mathrm{HG})$. Then, for every $\mathbf{v}_{h} \in X_{h}$ it holds that

$$
\left[p_{h}-p^{I}, \mathcal{D} \mathcal{I} \mathcal{V}_{\mathbf{v}_{h}}\right]_{Q_{h}} \lesssim h^{k+2}\|p\|_{k+3, \Omega}\left\|\mid \mathbf{v}_{h}\right\|_{X_{h}}
$$

Proof. We decompose the inner product as in (2.17), we note that $\mathcal{D} \mathcal{I} \mathcal{V} \mathbf{v}_{h}=\left(\widetilde{\mathcal{D I V} \mathbf{v}_{h}}\right)^{\mathrm{I}}$, we use $(2.19)$ and we note that $\widetilde{\mathcal{D I V} \mathbf{v}_{\left.h\right|_{E}}}$ is a polynomial of degree $k$ on $E$ to obtain:

$$
\left[p^{\mathrm{I}}, \mathcal{D} \mathcal{I} \mathcal{V} \mathbf{v}_{h}\right]_{Q_{h}}=\sum_{E \in \Omega_{h}}\left[p^{\mathrm{I}}, \mathcal{D} \mathcal{I} \mathcal{V} \mathbf{v}_{h}\right]_{E}=\sum_{E \in \Omega_{h}} \int_{E} p \widetilde{\mathcal{D I V} \mathbf{v}_{h}} \mathrm{~d} V
$$


We substitute (5.2a) above and integrate by parts on each cell, we sum over all the cells and note that $\mathbf{n}_{E, e}$. $R_{E}\left(\mathbf{v}_{h}\right)$ only depends on the degrees of freedom of edge/face $e$ and takes opposite signs on the two sides of each internal edge/face:

$$
\begin{aligned}
\sum_{E \in \Omega_{h}} \int_{E} p \widetilde{\mathcal{D \mathcal { I V }} \mathbf{v}_{h}} \mathrm{~d} V & =\sum_{E \in \Omega_{h}}\left(-\int_{E} \nabla p \cdot R_{E}\left(\mathbf{v}_{h}\right) \mathrm{d} V+\sum_{e \in \partial E} \int_{e} \mathbf{n}_{E, e} \cdot R_{E}\left(\mathbf{v}_{h}\right) p \mathrm{~d} S\right) \\
& =-\int_{\Omega} \nabla p \cdot R_{E}\left(\mathbf{v}_{h}\right) \mathrm{d} V+\sum_{e \in \mathcal{E}^{h} \cap \Gamma} \int_{e} \mathbf{n}_{E, e} \cdot R_{E}\left(\mathbf{v}_{h}\right) p \mathrm{~d} S .
\end{aligned}
$$

The last term above is further developed by using $(5.2 \mathrm{~b})$ and $(2.6)$ (with the boundary condition $g=p_{\left.\right|_{\Gamma}}$ ):

$$
\sum_{e \in \mathcal{E}^{h} \cap \Gamma} \int_{e} \mathbf{n}_{E, e} \cdot R_{E}\left(\mathbf{v}_{h}\right) p \mathrm{~d} S=\sum_{e \in \mathcal{E}^{h} \cap \Gamma} \int_{e} \widetilde{v}_{h}^{e} p \mathrm{~d} S=\left\langle p, \mathbf{v}_{h}\right\rangle_{h}
$$

Combining (5.6) and (5.7) in (5.5) yields

$$
\left[p^{\mathrm{I}}, \mathcal{D} \mathcal{I} \mathcal{V} \mathbf{v}_{h}\right]_{Q_{h}}=-\int_{\Omega} \nabla p \cdot R_{E}\left(\mathbf{v}_{h}\right) \mathrm{d} V+\left\langle p, \mathbf{v}_{h}\right\rangle_{h}
$$

Using scheme's equation (2.5a) and (5.8), we transform the left-hand side of (5.4) as follows

$$
\left[p_{h}-p^{\mathrm{I}}, \mathcal{D} \mathcal{I} \mathcal{V} \mathbf{v}_{h}\right]_{E}=\left[\mathbf{u}_{h}, \mathbf{v}_{h}\right]_{E}+\left\langle p, \mathbf{v}_{h}\right\rangle_{h}-\left[p^{\mathrm{I}}, \mathcal{D} \mathcal{I} \mathcal{V} \mathbf{v}_{h}\right]_{E}=\left[\mathbf{u}_{h}, \mathbf{v}_{h}\right]_{E}+\int_{\Omega} \nabla p \cdot R_{E}\left(\mathbf{v}_{h}\right) \mathrm{d} V
$$

We add and subtract $\nabla \widehat{p}$, where $\widehat{p}$ is the $(k+2)$-interpolant of $p$ as in (M4):

$$
\int_{E} \nabla p \cdot R_{E}\left(\mathbf{v}_{h}\right) \mathrm{d} V=\int_{E} \nabla \widehat{p} \cdot R_{E}\left(\mathbf{v}_{h}\right) \mathrm{d} V+\int_{E} \nabla(p-\widehat{p}) \cdot R_{E}\left(\mathbf{v}_{h}\right) \mathrm{d} V .
$$

We integrate by parts, use again (5.2a), (5.2b) and (2.24) to develop the first term in the right-hand side of (5.9):

$$
\begin{aligned}
\int_{E} \nabla \widehat{p} \cdot R_{E}\left(\mathbf{v}_{h}\right) \mathrm{d} V & =-\int_{E} \widehat{p} \operatorname{div} R_{E}\left(\mathbf{v}_{h}\right) \mathrm{d} V+\sum_{e \in \partial E} \int_{\partial e} \widehat{p} \mathbf{n}_{E, e} \cdot R_{E}\left(\mathbf{v}_{h}\right) \mathrm{d} S \\
& =-\int_{E} \widehat{p} \widetilde{\mathcal{D} \mathcal{I V} \mathbf{v}_{h}} \mathrm{~d} V+\sum_{e \in \partial E} \int_{e} \widehat{p} \widetilde{v}_{h} \mathrm{~d} S \\
& =\left[\left(\Pi_{k+1}^{E}(\mathrm{~K} \nabla \widehat{p})\right)^{\mathrm{I}}, \mathbf{v}_{h}\right]_{E}
\end{aligned}
$$

We substitute (5.10) in (5.9) and add and subtract $\mathbf{u}^{\mathrm{I}}$ to obtain the final expression:

$$
\begin{aligned}
\sum_{E}( & {\left.\left[\mathbf{u}_{h}, \mathbf{v}_{h}\right]_{E}+\int_{E} \nabla p \cdot R_{E}\left(\mathbf{v}_{h}\right) \mathrm{d} V\right) } \\
& =\sum_{E \in \Omega_{h}}\left(\left[\mathbf{u}_{h}-\mathbf{u}^{\mathrm{I}}, \mathbf{v}_{h}\right]_{E}+\left[\left(\mathbf{u}+\Pi_{k+1}^{E}(\mathrm{~K} \nabla \widehat{p})\right)^{\mathrm{I}}, \mathbf{v}_{h}\right]_{E}+\int_{E} \nabla(p-\widehat{p}) \cdot R_{E}\left(\mathbf{v}_{h}\right) \mathrm{d} V\right) \\
& =\mathrm{T}_{1}+\mathrm{T}_{2}+\mathrm{T}_{3} .
\end{aligned}
$$


To evaluate term $\mathrm{T}_{1}$, we apply the Cauchy-Schwarz inequality, definition (2.20) and the result of Theorem 4.1:

$$
\begin{aligned}
\mathrm{T}_{1} & \leq \sum_{E \in \Omega_{h}}\|\| \mathbf{u}_{h}-\mathbf{u}^{\mathrm{I}}\left\|\left.\right|_{E}\right\| \mathbf{v}_{h} \mid \|_{E} \leq\left(\sum_{E \in \Omega_{h}}\|\| \mathbf{u}_{h}-\mathbf{u}^{\mathrm{I}} \mid \|_{E}^{2}\right)^{1 / 2}\left(\sum_{E \in \Omega_{h}}\left\|\mathbf{v}_{h}\right\|_{E}^{2}\right)^{1 / 2} \\
& =\left.\left\|\left|\mathbf{u}_{h}-\mathbf{u}^{\mathrm{I}}\right|\right\|_{X_{h}}\left\|\mathbf{v}_{h}\right\|\right|_{X_{h}} \lesssim h^{k+2}\|p\|_{k+3, \Omega}\left\|\mathbf{v}_{h} \mid\right\|_{X_{h}} .
\end{aligned}
$$

To evaluate term $\mathbf{T}_{2}$, we apply the Cauchy-Schwarz inequality, definition (2.20), substitute $\mathbf{u}=-\mathrm{K} \nabla p$, and apply Lemma 3.4:

$$
\begin{aligned}
\mathrm{T}_{2} & \leq \sum_{E \in \Omega_{h}}\|\|\left(\mathbf{u}+\Pi_{k+1}^{E}(\mathrm{~K} \nabla \widehat{p})\right)^{\mathrm{I}}\|\|_{E}\left\|\left|\mathbf{v}_{h}\right|\right\|_{E} \\
& \leq\left(\sum_{E \in \Omega_{h}}\|\|\left(-\mathrm{K} \nabla p+\Pi_{k+1}^{E}(\mathrm{~K} \nabla \widehat{p})\right)^{\mathrm{I}}\|\|_{E}^{2}\right)^{1 / 2}\left(\sum_{E \in \Omega_{h}}\left\|\mathbf{v}_{h}\right\| \|_{E}^{2}\right)^{1 / 2} \\
& =\left(\sum_{E \in \Omega_{h}} h_{E}^{2(k+2)}\|p\|_{k+3, E}^{2}\right)^{1 / 2}\left\|\mathbf{v}_{h}\left|\left\|_{X_{h}} \lesssim h^{k+2}\right\| p\left\|_{k+3, \Omega}\right\| \mathbf{v}_{h}\right|\right\|_{X_{h}} .
\end{aligned}
$$

To evaluate term $T_{3}$, we apply the Cauchy-Schwarz inequality twice, the error estimate in (M4) and the equivalence in (5.3):

$$
\begin{aligned}
\mathrm{T}_{3} & \leq \sum_{E \in \Omega_{h}}\|\nabla(p-\widehat{p})\|_{0, E}\left\|R_{E}\left(\mathbf{v}_{h}\right)\right\|_{0, E} \leq\left(\sum_{E \in \Omega_{h}}\|\nabla(p-\widehat{p})\|_{0, E}^{2}\right)^{1 / 2}\left(\sum_{E \in \Omega_{h}}\left\|R_{E}\left(\mathbf{v}_{h}\right)\right\|_{0, E}^{2}\right)^{1 / 2} \\
& \lesssim\left(\sum_{E \in \Omega_{h}} h_{E}^{2(k+2)}|p|_{k+3, E}^{2}\right)^{1 / 2}\left(\sum_{E \in \Omega_{h}}\left\|\mid \mathbf{v}_{h}\right\|_{E}^{2}\right)^{1 / 2} \lesssim h^{k+2}\|p\|_{k+3, \Omega}\left\|\mathbf{v}_{h}\right\| \|_{X_{h}} .
\end{aligned}
$$

The assertion of the lemma follows by combining the estimates for $T_{1}, T_{2}$, and $T_{3}$ in (5.11).

\subsection{Discrete inf-sup condition}

Lemma 5.5. For any scalar grid function $q_{h} \in Q_{h}$, there exists a vector grid function $\mathbf{v}_{h}^{q} \in X_{h}$ such that

$$
\begin{aligned}
{\left[\mathcal{D} \mathcal{I} \mathcal{V} \mathbf{v}_{h}^{q}, q_{h}\right]_{Q_{h}} } & =\left\|q_{h}\right\|_{Q_{h}}^{2} \\
\|\left.\left|\mathbf{v}_{h}^{q}\right|\right|_{X_{h}} & \left.\lesssim\left\|q_{h}\right\|\right|_{Q_{h}}
\end{aligned}
$$

Proof. The inf-sup condition can be proved by adapting a similar proof from [45]. Consider $q_{h} \in Q_{h}$ and $\widetilde{q}_{h} \in L^{2}(\Omega)$, which is the piecewise $k$-degree polynomial on $\Omega_{h}$ given by $(2.14)$. We recall that $\left(\widetilde{q}_{h}\right)^{\mathrm{I}}=q_{h}$ and that $\left\|\widetilde{q}_{h}\right\|_{0, \Omega}=\left\|\left|q_{h}\right|\right\|_{Q_{h}}$. Consider a ball $B$ that contains domain $\Omega$ and the function $\widetilde{q}_{h}^{\text {ext }}$ that extends $\widetilde{q}_{h}$ by zero in the region $B \backslash \Omega$. Now, let $\psi \in H^{2}(B)$ be the solution of $\Delta \psi=\widetilde{q}_{h}^{\text {ext }}$ in $B$ with homogeneous conditions on the boundary $\partial B$. Since $B$ is $H^{2}$-regular and $\widetilde{q}_{h}^{\text {ext }}$ is zero outside $\Omega$, we have that

$$
\|\psi\|_{2, \Omega} \leq\|\psi\|_{2, B} \leq C_{B}^{*}\left\|\widetilde{q}_{h}^{e x t}\right\|_{0, B}=C_{B}^{*}\left\|\widetilde{q}_{h}\right\|_{0, \Omega}=C_{B}^{*}\left\|q_{h} \mid\right\|_{Q_{h}},
$$

where constant $C_{B}^{*}$ is a positive constant independent of $\psi$ and $h$. Since $\psi \in H^{2}(B)$ we have that $\nabla \psi \in\left(H^{1}(B)\right)^{d}$ and $\nabla \psi_{\left.\right|_{\Omega}} \in\left(H^{1}(\Omega)\right)^{d} \subset V_{\Omega_{h}}$, and, thus, the interpolant of $\nabla \psi$ in $X_{h}$ is well-defined. Take $\mathbf{v}_{h}^{q}=(\nabla \psi)^{\mathrm{I}}$. We immediately have that

$$
\mathcal{D I} \mathcal{V} \mathbf{v}_{h}^{q}=\mathcal{D} \mathcal{I} \mathcal{V}(\nabla \psi)^{\mathrm{I}}=(\Delta \psi)^{\mathrm{I}}=\left(\widetilde{q}_{h}\right)^{\mathrm{I}}=q_{h},
$$


which proves (5.12a). Then, Lemma 3.3 implies that

$$
\left\|\left|\mathbf{v}_{h}^{q}\right|\right\|_{E}^{2}=\left\|\left.\left|(\nabla \psi)^{\mathrm{I}}\right|\right|_{E} ^{2} \lesssim\right\| \nabla \psi\left\|_{0, E}^{2}+h_{E}^{2}|\nabla \psi|_{1, E}^{2} \lesssim\right\| \psi \|_{2, E}^{2} .
$$

Summing over all $E \in \Omega_{h}$ and using the $H^{2}$-regularity inequality (5.13) prove (5.12b):

$$
\left\|\mid \mathbf{v}_{h}^{q}\right\|_{X_{h}}^{2}=\sum_{E \in \Omega_{h}}\|\| \mathbf{v}_{h}^{q}\left\|_{E}^{2} \lesssim \sum_{E \in \Omega_{h}}\right\| \psi\left\|_{2, E}^{2} \lesssim\right\| \psi\left\|_{2, \Omega}^{2} \lesssim\right\| \widetilde{q}_{h}\left\|_{0, \Omega}^{2} \lesssim\right\| q_{h} \|_{Q_{h}}^{2}
$$

This concludes the proof of the Lemma.

Now, consider the mesh-dependent norm in $X_{h}$ defined as $\left|\left\|\mathbf{v}_{h}\left|\left\|_{\mathcal{D I V}}^{2}=\right\|\right| \mathbf{v}_{h}||_{X_{h}}^{2}+\right\| \mathcal{D} \mathcal{I} \mathcal{V} \mathbf{v}_{h}\right| \|_{Q_{h}}^{2}$. If $\mathbf{v}_{h}^{q}$ is the flux associated with $q_{h}$ through the proof of Lemma 5.5 we know that $\mathcal{D} \mathcal{I} \mathcal{V} \mathbf{v}_{h}^{q}=q_{h}$. We add $\left.\left\|\mathcal{D} \mathcal{I} \mathcal{V} \mathbf{v}_{h}^{q}\right\|\right|_{Q_{h}}=$ $\left\|q_{h} \mid\right\|_{Q_{h}}$ to both sides of (5.12b) and we have that $\left.\left\|\mathbf{v}_{h}^{q}\right\|\left\|_{\mathcal{D I V}} \lesssim\right\|\left\|q_{h}\right\|\right|_{Q_{h}}$. Using this inequality and both (5.12a) and $(5.12 b)$ we immediately find that

$$
\sup _{\mathbf{v}_{h} \in X_{h} \backslash\{0\}} \frac{\left[\mathcal{D} \mathcal{I} \mathcal{V} \mathbf{v}_{h}, q_{h}\right]_{Q_{h}}}{\left\|\left|\mathbf{v}_{h}\right|\right\|_{\mathcal{D I V}}} \geq \frac{\left[\mathcal{D} \mathcal{I} \mathcal{V} \mathbf{v}_{h}^{q}, q_{h}\right]_{Q_{h}}}{\left\|\mathbf{v}_{h}^{q}\right\|_{\mathcal{D I V}}}=\frac{\left\|q_{h} \mid\right\|_{Q_{h}}^{2}}{\left\|\mathbf{v}_{h}^{q}\right\|_{\mathcal{D I V}}} \gtrsim\left\|q_{h}\right\| \|_{Q_{h}} .
$$

Dividing both sides by $\left\|q_{h}\right\| \|_{Q_{h}}$ and taking the infimum on $q_{h} \in Q_{h}$ gives the standard form for the discrete inf-sup inequality. We formally state this result, which can be seen as a corollary of Lemma 5.5, as follows by introducing the "inf-sup constant" $\beta_{*}>0$.

Corollary 5.6. There exists a strictly positive constant $\beta_{*}$, which is independent of $h$, such that

$$
\inf _{q_{h} \in Q_{h} \backslash\{0\}} \sup _{\mathbf{v}_{h} \in X_{h} \backslash\{0\}} \frac{\left[\mathcal{D} \mathcal{I} \mathcal{V} \mathbf{v}_{h}, q_{h}\right]_{Q_{h}}}{\left\|| \mathbf { v } _ { h } | | _ { \mathcal { D I V } } \left|\left\|q_{h} \mid\right\|_{Q_{h}}\right.\right.} \geq \beta_{*} .
$$

\subsection{Proof of Theorem 5.1}

Let $\mathbf{v}_{h}^{q} \in X_{h}$ be the flux corresponding to $q_{h}=p_{h}-p^{\mathrm{I}}$ in the discrete inf-sup condition of Lemma 5.5. Using Lemmas 5.4 and 5.5 we have that

$$
\left.\left\||| p_{h}-p^{\mathrm{I}}\right\|\right|_{Q_{h}} ^{2}=\left[p_{h}-p^{\mathrm{I}}, \mathcal{D} \mathcal{I} \mathcal{V} \mathbf{v}_{h}^{q}\right]_{Q_{h}} \lesssim h^{k+2}\|p\|_{k+3, \Omega}\left|\left\|\mathbf{v}_{h}^{q}\right\|\right|_{X_{h}} \lesssim h^{k+2}\|p\|_{k+3, \Omega}\left\|\mid p_{h}-p^{\mathrm{I}}\right\| \|_{Q_{h}},
$$

which implies the assertion of Theorem 5.1.

\subsection{Error estimates for the polynomial approximation of the scalar unknown}

According to Remark 2.4, we can compute the polynomial $\widetilde{p}_{h}$ inside each cell $E$ through (2.14) by using only the degrees of freedom of the mimetic solution $p_{h}$ pertinent to that cell. This polynomial provides a pointwise approximation of the exact solution $p$ for $k \geq 0$ and its gradient $\nabla p$ for $k>0$. The accuracy of such an approximation is stated in the following theorem.

Theorem 5.7. Let $k$ be a non-negative integer number and $p \in H^{k+3}(\Omega)$ the exact solution of problem (2.1a)(2.1c). Let $p_{h} \in Q_{h}$ be the solution of (2.5a) and (2.5b) under assumption (HG), and $\widetilde{p}_{h}$ the piecewise polynomial function that in each cell $E$ is given by (2.14). Then, it holds:

$$
\left\|p-\widetilde{p}_{h}\right\|_{0, \Omega}+h\left|p-\widetilde{p}_{h}\right|_{1, \Omega} \lesssim h^{k+1}\|p\|_{k+3, \Omega} .
$$


Proof. Adding and subtracting $\widetilde{p_{E}^{I}}$ and using the triangle inequality yield:

$$
\left\|p-\widetilde{p}_{h}\right\|_{0, \Omega}^{2}=\sum_{E \in \Omega_{h}}\left\|p-\widetilde{p}_{h}\right\|_{0, E}^{2} \lesssim \sum_{E \in \Omega_{h}}\left(\left\|p-\widetilde{p_{E}^{I}}\right\|_{0, E}^{2}+\left\|\widetilde{p_{E}^{I}}-\widetilde{p}_{h}\right\|_{0, E}^{2}\right) .
$$

Consider cell $E$. As noted in Remark 2.4, we have that $\widetilde{p_{E}^{I}}=\Pi_{k}^{E}(p)$. Using the estimate for the projection operator yields

$$
\left\|p-\widetilde{p_{E}^{I}}\right\|_{0, E}=\left\|p-\Pi_{k}^{E}(p)\right\|_{0, E} \lesssim h_{E}^{k+1}\|p\|_{k+1, E}
$$

so that

$$
\sum_{E \in \Omega_{h}}\left\|p-\widetilde{p_{E}^{I}}\right\|_{0, E}^{2} \lesssim h^{2(k+1)} \sum_{E \in \Omega_{h}}\|p\|_{k+1, E}^{2} \lesssim h^{2(k+1)}\|p\|_{k+1, \Omega}^{2}
$$

The second term can be estimated by first noting that

$$
\left\|\widetilde{p_{E}^{\mathrm{I}}}-\widetilde{p}_{h}\right\|_{0, E}^{2}=\left\|\left(\widetilde{p^{\mathrm{I}}-p_{h}}\right)\right\|_{0, E}^{2}=\left|\left\|p^{\mathrm{I}}-p_{h} \mid\right\|_{E}^{2}\right.
$$

and, then, using the result of Theorem 5.1:

$$
\sum_{E \in \Omega_{h}}\left\|\widetilde{p_{E}^{I}}-\widetilde{p}_{h}\right\|_{0, E}^{2}=\sum_{E \in \Omega_{h}}\left\|\left|p^{I}-p_{h}\|\|_{E}^{2}=\left\|\mid p^{I}-p_{h}\right\|\left\|_{Q_{h}}^{2} \lesssim h^{2(k+2)}\right\| p \|_{k+3, \Omega}^{2} .\right.\right.
$$

An $\mathcal{O}\left(h^{k}\right)$ estimate can be derived for $\left|p-\widetilde{p}_{h}\right|_{1, \Omega}$ by repeating the same argument since a standard inverse inequality implies that

$$
\left.\left\|\nabla\left(\widetilde{p^{\mathrm{I}}-p_{h}}\right)\right\|_{0, E} \lesssim h_{E}^{-1} \| \widetilde{\left(p^{\mathrm{I}}-p_{h}\right.}\right)\left\|_{0, E} \lesssim h_{E}^{-1}\right\|\left\|p^{\mathrm{I}}-p_{h}\right\| \|_{E}
$$

The assertion of the theorem follows by combining together these estimates.

Remark 5.8. The piecewise polynomial approximation offered by $\widetilde{p}_{h}$ does not use the information provided by the flux approximation $\mathbf{u}_{h}$. A better pointwise approximation to the exact solution $p$ and its gradients could be given by devising some special post-processing technique as was done for the low-order MFD method in [22] and for the lowest-order version of this method (i.e., for $k=0$ ) in $[8,12]$. Post-processing for arbitrary $k$ is currently under development and will be the content of a future paper.

\section{Numerical EXPERIMENTS}

The numerical experiments presented in this section are aimed to confirm the a priori analysis of Sections 4 and 5 . We consider here only the case $d=2$; for $d=3$ we expect to see the same behavior. In a preliminary stage, the consistency of the method i.e., the exactness of these methods for polynomial solutions, has been tested numerically by solving problem (2.1a)-(2.1c) with boundary and source data determined by $p(x, y)=x^{m}+y^{m}$ on different set of polygonal meshes and for $m=1$ to 5 . In all the cases, the method based on polynomials of degree $k \geq m$ provided errors whose magnitude was of the order of the arithmetic precision, thus confirming this property. These results are not reported here.

To study the accuracy of the method we solve the diffusion equation on the domain $\Omega=] 0,1[\times] 0,1[$ using the variable diffusion coefficient:

$$
\mathbf{K}(x, y)=e^{x+y} \mathbf{I}+\left(\begin{array}{cc}
1+y^{2} & -x y \\
-x y & 1+x^{2}
\end{array}\right)
$$




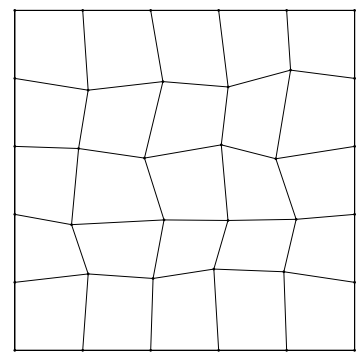

$\left(\mathcal{M}_{1}\right)$

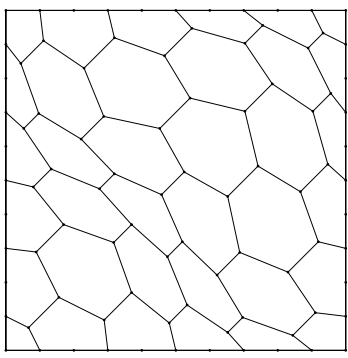

$\left(\mathcal{M}_{2}\right)$

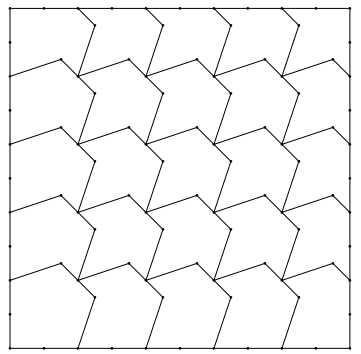

$\left(\mathcal{M}_{3}\right)$

Figure 2. The first mesh of mesh families $\mathcal{M}_{1}$ (left panel), $\mathcal{M}_{2}$ (middle panel), $\mathcal{M}_{3}$ (right panel) that are used in the convergence and comparison tests of the numerical experiment section. The mesh cells in $\mathcal{M}_{1}$ are randomized quadrilaterals. The mesh cells in $\mathcal{M}_{2}$ are mainly smoothly remapped hexagons: two quadrilateral cells close the domain at opposite bottomleft and top-right-corners, while all the other cells are hexagons with possible degeneracy (two parallel edges) on the domain boundary. The mesh cells in $\mathcal{M}_{3}$ are regular non-convex octagons, cut at right and top boundaries to fit into the unit square domain.

The forcing term in $(2.1 \mathrm{~b})$ and the Dirichlet boundary condition on $\partial \Omega$ are set in accordance with the exact solution:

$$
p(x, y)=\mathrm{e}^{-2 \pi y} \sin (2 \pi x)+\cos (2 \pi(x+2 y)) .
$$

The performance of the mixed mimetic method are investigated by evaluating the rate of convergence on three different sequences of five meshes, labeled by $\mathcal{M}_{1}, \mathcal{M}_{2}$, and $\mathcal{M}_{3}$, respectively. Figures $2 \mathrm{a}-2 \mathrm{c}$ show the first mesh of each sequence. The meshes in $\mathcal{M}_{1}$ are built by partitioning the domain $\Omega$ into square cells and relocating each interior node to a random position inside a square box centered at that node. The sides of this square box are aligned with the coordinate axis and their lenght is equal to 0.8 times the minimum distance between two adjacent nodes of the initial square mesh.

The meshes in $\mathcal{M}_{2}$ are built as follows. First, we determine a primal mesh by remapping the position $(\widehat{x}, \widehat{y})$ of the nodes of an uniform square partition of $\Omega$ by the smooth coordinate transformation (see, for example, [19]):

$$
\begin{aligned}
& x=\widehat{x}+0.1 \sin (2 \pi \widehat{x}) \sin (2 \pi \widehat{y}), \\
& y=\widehat{y}+0.1 \sin (2 \pi \widehat{x}) \sin (2 \pi \widehat{y}) .
\end{aligned}
$$

The corresponding mesh of $\mathcal{M}_{2}$ is built from the primal mesh by splitting each quadrilateral cell into two triangles and connecting the barycenters of adjacent triangular cells by a straight segment. The mesh construction is completed at the boundary by connecting the barycenters of the triangular cells close to the boundary to the midpoints of the boundary edges and these latters to the boundary vertices of the primal mesh.

The meshes in $\mathcal{M}_{3}$ are obtained by filling the unit square with a suitably scaled non-convex octagonal reference cell, which is cut at the right and top domain boundaries to fit the unit square domain $\Omega$.

All the meshes are parametrized by the number of partitions in each direction. The starting mesh of every sequence is built from a $5 \times 5$ regular grid, and the refined meshes are obtained by doubling this resolution.

For each calculation we consider the relative errors

$$
\mathcal{E}_{X_{h}}=\frac{\|\| \mathbf{u}^{\mathrm{I}}-\mathbf{u}_{h} \mid \|_{X_{h}}}{\left\|\mathbf{u}^{\mathrm{I}} \mid\right\|_{X_{h}}}, \quad \mathcal{E}_{Q_{h}}=\frac{\left\|p^{\mathrm{I}}-p_{h}\right\| \|_{Q_{h}}}{\left\|\left|\left\|p^{\mathrm{I}} \mid\right\|_{Q_{h}}\right.\right.}, \quad \mathcal{E}_{L^{2}}=\frac{\left\|p-\widetilde{p}_{h}\right\|_{0, \Omega}}{\|p\|_{0, \Omega}}, \quad \mathcal{E}_{H^{1}}=\frac{\left|p-\widetilde{p}_{h}\right|_{1, \Omega}}{|p|_{1, \Omega}} .
$$



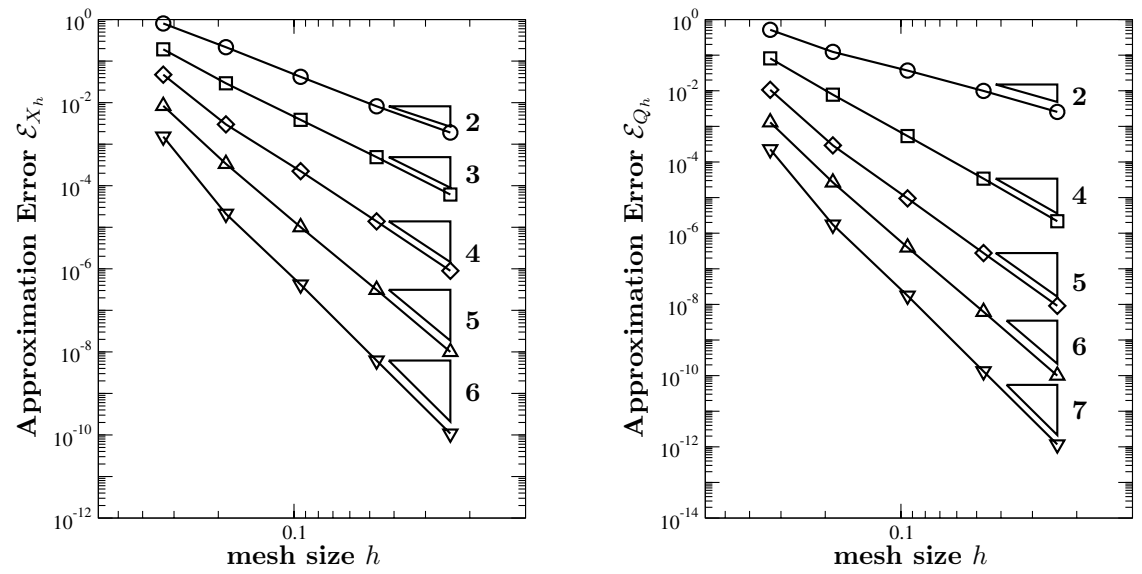

FiguRE 3. Error curves $\mathcal{E}_{X_{h}}$ (left plot) and $\mathcal{E}_{Q_{h}}$ (right plot) with respect to the mesh size $h$ using mesh family $\mathcal{M}_{1}$ of randomized quadrilaterals. The MFD method uses polynomials of degree $k=0$ (circles), $k=1$ (squares), $k=2$ (diamonds), $k=3$ (up triangles), $k=4$ (down triangles). Slopes proportional to $h^{k+2}$ are shown in the left plot. As a superconvergence effect is present in the scalar approximation, for $k \geq 1$, in the right plot we show the slope for $h^{2}$ $(k=0)$ and the slopes proportional to $h^{k+3}$ for $k \geq 1$.
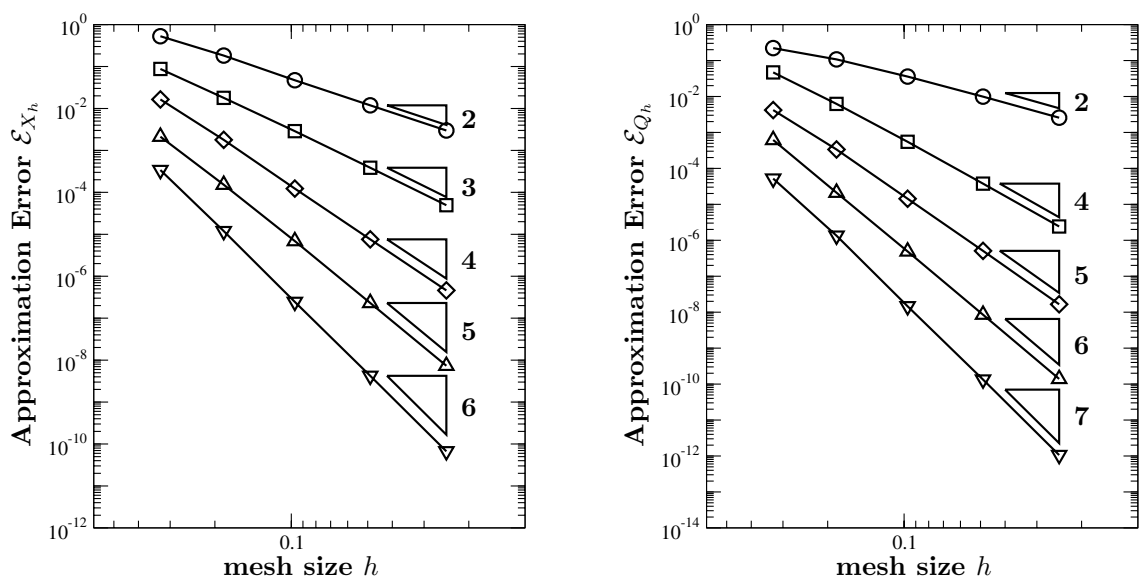

FIGURE 4. Error curves $\mathcal{E}_{X_{h}}$ (left plot) and $\mathcal{E}_{Q_{h}}$ (right plot) with respect to the mesh size $h$ using mesh family $\mathcal{M}_{2}$ of smoothly remapped hexagons. The MFD method uses polynomials of degree $k=0$ (circles), $k=1$ (squares), $k=2$ (diamonds), $k=3$ (up triangles), $k=4$ (down triangles). Slopes proportional to $h^{k+2}$ are shown in the left plot. As a superconvergence effect is present in the scalar approximation, for $k \geq 1$, in the right plot we show the slope for $h^{2}$ $(k=0)$ and the slopes proportional to $h^{k+3}$ for $k \geq 1$.

Errors $\mathcal{E}_{X_{h}}$ and $\mathcal{E}_{Q_{h}}$ are both expected to decrease to zero proportionally to $\mathcal{O}\left(h^{k+2}\right)$ in accordance with Theorems 4.1 and 5.1. Errors $\mathcal{E}_{L^{2}}$ and $\mathcal{E}_{H^{1}}$ are expected to decrease to zero proportionally to $\mathcal{O}\left(h^{k+1}\right)$ and $\mathcal{O}\left(h^{k}\right)$, respectively, in accordance with Theorem 5.7.

In Figures 3-5 we report the log-log plots of $\mathcal{E}_{X_{h}}$ (left plots) and $\mathcal{E}_{Q_{h}}$ (right plots) versus the mesh size parameter $h$ for calculations using mesh families $\mathcal{M}_{1}, \mathcal{M}_{2}$, and $\mathcal{M}_{3}$. In Figure 6 we report the $\log$-log plots of $\mathcal{E}_{L^{2}}$ and $\mathcal{E}_{H^{1}}$ versus the mesh size parameter $h$ for calculations using the meshes in $\mathcal{M}_{1}$. All calculations were 

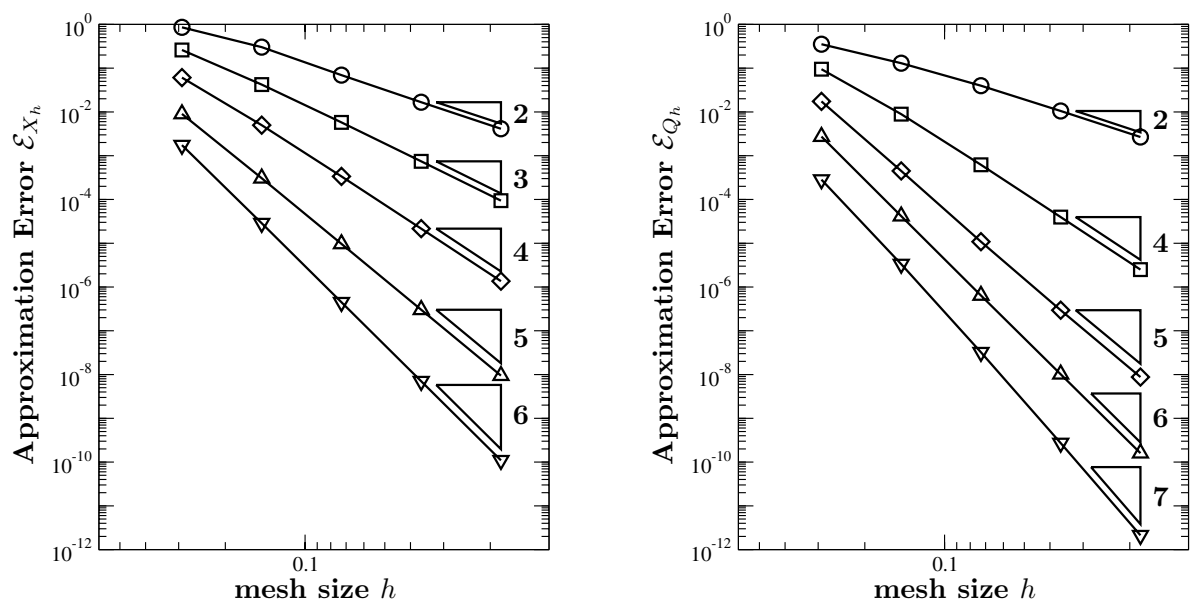

FiguRE 5. Error curves $\mathcal{E}_{X_{h}}$ (left plot) and $\mathcal{E}_{Q_{h}}$ (right plot) with respect to the mesh size $h$ using mesh family $\mathcal{M}_{3}$ of regular non-convex octagons. The MFD method uses polynomials of degree $k=0$ (circles), $k=1$ (squares), $k=2$ (diamonds), $k=3$ (up triangles), $k=4$ (down triangles). Slopes proportional to $h^{k+2}$ are shown in the left plot. As a superconvergence effect is present in the scalar approximation, for $k \geq 1$, in the right plot we show the slope for $h^{2}$ $(k=0)$ and the slopes proportional to $h^{k+3}$ for $k \geq 1$.
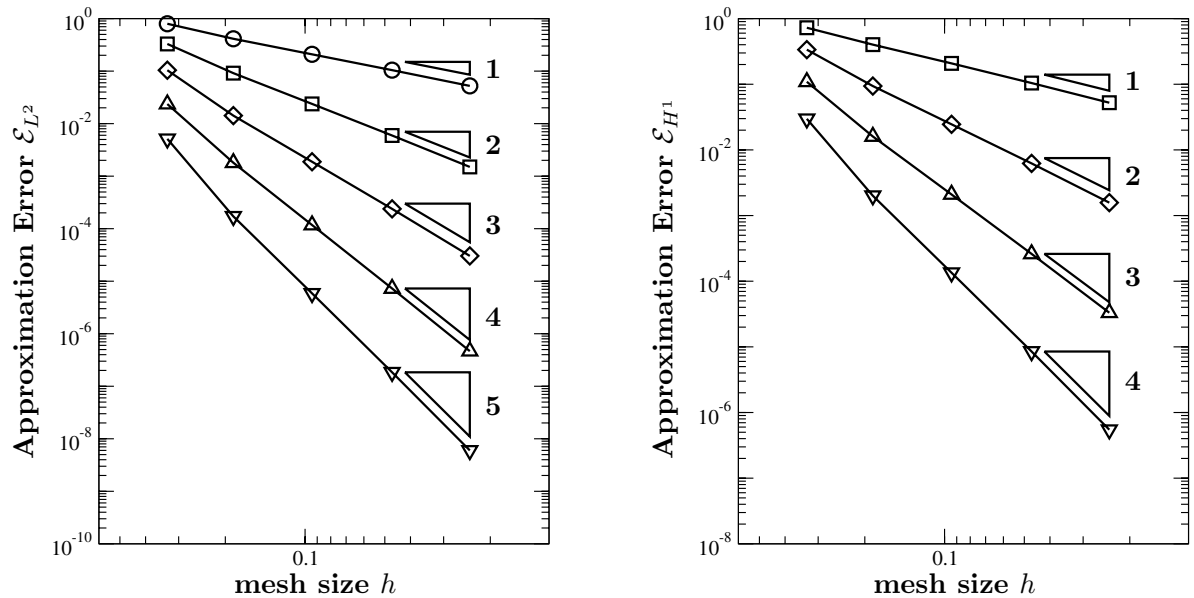

FiguRE 6. Error curves $\mathcal{E}_{L^{2}}$ (left plot) and $\mathcal{E}_{H^{1}}$ (right plot) with respect to the mesh size $h$ using mesh family $\mathcal{M}_{1}$ of randomized quadrilaterals. The MFD method uses polynomials of degree $k=0$ (circles), $k=1$ (squares), $k=2$ (diamonds), $k=3$ (up triangles), $k=4$ (down triangles). Slopes proportional to $h^{k+1}$ and $h^{k}$ are shown in the left and right plot, respectively. The error curves for the gradient approximation measured by $\mathcal{E}_{H^{1}}$ starts at $k=1$.

carried out by using the mixed MFD method based on polynomials of degree from $k=0$ to 4 as indicated in the captions.

The convergence rates are reflected by the slope of each error curve, which has to be compared with the exact slope shown in the plot by a triangle closed to each curve and indicated by the corresponding integer number. The rates observed experimentally for the flux approximation are always in good agreement with the 
estimate of Theorem 4.1 and the errors measured in the $X_{h}$-norm decrease proportionally to $h^{k+2}$. Also, the approximation of the exact solution and its gradient that are provided by the piecewise polynomial function $\widetilde{p}_{h}$ and $\nabla \widetilde{p}_{h}$ behaves as expected from Theorem 5.7, and the errors measured in the $L^{2}$ - and energy- norm decrease proportionally to $h^{k+1}$ and $h^{k}$. We show these error plots only for the calculations using $\mathcal{M}_{1}$ as for the other meshes the behavior is the same. Instead, the approximation error $\mathcal{E}_{Q_{h}}$ behaves as stated by Theorem 5.1 only for the lowest-order accurate scheme, i.e., for $k=0$, where error $\mathcal{E}_{Q_{h}}$ decreases like $\mathcal{O}\left(h^{2}\right)$. For $k \geq 1$, an ultraconvergence effect is visible for the three mesh families $\mathcal{M}_{1}, \mathcal{M}_{2}, \mathcal{M}_{3}$, as error $\mathcal{E}_{Q_{h}}$ decreases like $\mathcal{O}\left(h^{k+3}\right)$ instead of $\mathcal{O}\left(h^{k+2}\right)$ as we would expect from Theorem 5.1. An extensive validation on different kind of meshes, exact solutions and constant and variable diffusion tensors (results are not reported here) confirms that this effect seems to persist, and, therefore, to be quite general. Currently, we do not have a theoretical explanation of this phenomenon even if we may conjecture a connection with the nature of the degrees of freedom of the scalar variable $p_{h}$. In fact, error $\mathcal{E}_{Q_{h}}$ is a straightforward measure of how well the polynomial moments $p^{\mathrm{I}}$ of the exact solution $p$ are approximated by the degrees of freedom of the mimetic approximation $p_{h}$.

\section{Conclusions}

We presented the new family of mimetic finite difference schemes that extends to arbitrary order of accuracy the approximation of the scalar unknown and the flux of [19]. The well-posedness of the method and the convergence of the approximation are proved theoretically and convergence estimates for both the scalar and the flux variable are derived. The behavior of the method in solving diffusion problems with variable diffusion tensor is investigated experimentally and the numerical results confirm the convergence rates that are expected from the theory. An ultraconvergence effects is visible for the scalar variable when the error is measured in the mesh-dependent norm induced by the mimetic inner product for scalar functions. A complete understanding of this phenomenon will be the topic of a future work.

Acknowledgements. This work was partially supported by the National Nuclear Security Administration of the U.S. Department of Energy at Los Alamos National Laboratory under Contract No. DE-AC52-06NA25396 and the DOE Office of Science Advanced Scientific Computing Research (ASCR) Program in Applied Mathematics.

\section{REFERENCES}

[1] I. Aavatsmark, T. Barkve, O. Bœ and T. Mannseth, Discretization on unstructured grids for inhomogeneous, anisotropic media. Part i: Derivation of the methods. SIAM J. Sci. Comput. 19 (1998) 1700-1716.

[2] I. Aavatsmark, T. Barkve, O. Bœ and T. Mannseth, Discretization on unstructured grids for inhomogeneous, anisotropic media. Part ii: Discussion and numerical results. SIAM J. Sci. Comput. 19 (1998) 1717-1736.

[3] P. Antonietti, L. Beirão da Veiga, N. Bigoni and M. Verani, Mimetic finite differences for nonlinear and control problems. Math. Models Methods Appl. Sci. 24 (2014) 1457-1493.

[4] L. Beirão da Veiga, A mimetic discretization method for linear elasticity. ESAIM: M2AN 44 (2010) 231-250.

[5] L. Beirão da Veiga, F. Brezzi, A. Cangiani, G. Manzini, L.D. Marini and A. Russo, Basic principles of virtual element methods. Math. Models Methods Appl. Sci. 23 (2013) 119-214.

[6] L. Beirão da Veiga, J. Droniou and G. Manzini, A unified approach to handle convection term in finite volumes and mimetic discretization methods for elliptic problems. IMA J. Num. Anal. 31 (2011) 1357-1401.

[7] L. Beirão da Veiga, V. Gyrya, K. Lipnikov and G. Manzini, Mimetic finite difference method for the Stokes problem on polygonal meshes. J. Comput. Phys. 228 (2009) 7215-7232.

[8] L. Beirão da Veiga, K. Lipnikov and G. Manzini, Convergence analysis of the high-order mimetic finite difference method. Numer. Math. 113 (2009) 325-356.

[9] L. Beirão da Veiga, K. Lipnikov and G. Manzini, Arbitrary-order nodal mimetic discretizations of elliptic problems on polygonal meshes. SIAM J. Numer. Anal. 49 (2011) 1737-1760.

[10] L. Beirão da Veiga, K. Lipnikov and G. Manzini, Error analysis for a mimetic discretization of the steady Stokes problem on polyhedral meshes. SIAM J. Numer. Anal. 48 (2011) 1419-1443.

[11] L. Beirão da Veiga, K. Lipnikov and G. Manzini, The Mimetic Finite Difference Method. Vol. 11 of Model. Simul. Appl. 1st edition. Springer-Verlag, New York (2014).

[12] L. Beirão da Veiga and G. Manzini, A higher-order formulation of the mimetic finite difference method. SIAM J. Sci. Comput. 31 (2008) 732-760. 
[13] L. Beirão da Veiga and D. Mora, A mimetic discretization of the Reissner-Mindlin plate bending problem. Numer. Math. 117 (2011) 425-462.

[14] P. Bochev and J.M. Hyman, Principle of mimetic discretizations of differential operators. Compatible discretizations. In Proc. of IMA hot topics workshop on compatible discretizations, edited by D. Arnold, P. Bochev, R. Lehoucq, R. Nicolaides and M. Shashkov. IMA. Springer-Verlag 142 (2006) 89-120.

[15] D. Boffi, F. Brezzi and M. Fortin, Mixed finite element methods and applications. Springer Series Comput. Math. Springer, Berlin, Heidelberg (2013).

[16] S. Brenner and L. Scott, The Mathematical Theory of Finite Element Methods. Springer-Verlag, Berlin/Heidelberg (1994).

[17] F. Brezzi, A. Buffa and K. Lipnikov, Mimetic finite differences for elliptic problems. ESAIM: M2AN 43 (2009) $277-295$.

[18] F. Brezzi, A. Buffa and G. Manzini, Mimetic inner products for discrete differential forms. J. Comput. Phys. B 257 (2014) $1228-1259$.

[19] F. Brezzi, K. Lipnikov and M. Shashkov, Convergence of the mimetic finite difference method for diffusion problems on polyhedral meshes. SIAM J. Numer. Anal. 43 (2005) 1872-1896.

[20] J. Campbell and M. Shashkov, A tensor artificial viscosity using a mimetic finite difference algorithm. J. Comput. Phys. 172 (2001) 739-765.

[21] A. Cangiani, F. Gardini and G. Manzini, Convergence of the mimetic finite difference method for eigenvalue problems in mixed form. Comput. Methods Appl. Mech. Engrg. 200 (2011) 1150-1160.

[22] A. Cangiani and G. Manzini, Flux reconstruction and pressure post-processing in mimetic finite difference methods. Comput. Methods Appl. Mech. Engrg. 197 (2008) 933-945.

[23] B. Cockburn, J. Gopalakrishnan and R. Lazarov, Unified hybridization of discontinuous Galerkin, mixed, and continuous Galerkin methods for second order elliptic problems. SIAM J. Numer. Anal. 47 (2009) 1319-1365.

[24] Y. Coudière and G. Manzini, The discrete duality finite volume method for convection-diffusion problems. SIAM J. Numer. Anal. 47 (2010) 4163-4192.

[25] D.A. Di Pietro and A. Ern, Mathematical Aspects of Discontinuous Galerkin Methods. Mathématiques et Applications. Springer (2011).

[26] D.A. Di Pietro and A. Ern, Arbitrary-order mixed methods for heterogeneous anisotropic diffusion on general meshes, hal-00918482-v3 (2013).

[27] D.A. Di Pietro and A. Ern, Hybrid high-order methods for variable diffusion problems on general meshes. C. R. Math. 353 (2014) 31-34.

[28] K. Domelevo and P. Omnes, A finite volume method for the Laplace equation on almost arbitrary two-dimensional grids. ESAIM: M2AN 39 (2005) 1203-1249.

[29] J. Droniou, Finite volume schemes for diffusion equations: introduction to and review of modern methods. Math. Models Methods Appl. Sci. 24 (2014) 1575-1619.

[30] J. Droniou, R. Eymard, T. Gallouët and R. Herbin, A unified approach to mimetic finite difference, hybrid finite volume and mixed finite volume methods. Math. Models Methods Appl. Sci. 20 (2010) 265-295.

[31] J. Droniou, R. Eymard, T. Gallouët and R. Herbin, Gradient schemes: a generic framework for the discretisation of linear, nonlinear and nonlocal elliptic and parabolic equations. Math. Models Methods Appl. Sci. 23 (2013) 2395-2432.

[32] R. Eymard, T. Gallouët and R. Herbin, The finite volume method. In Handbook for Numerical Analysis, edited by P. Ciarlet and J.L. Lions. North Holland (2000) 715-1022.

[33] R. Eymard, T. Gallouet and R. Herbin, Discretization of heterogeneous and anisotropic diffusion problems on general nonconforming meshes. SUSHI: a scheme using stabilization and hybrid interface. IMA J. Numer. Anal. 30 (2010) 1009-1043.

[34] V. Gyrya and K. Lipnikov, High-order mimetic finite difference method for diffusion problems on polygonal meshes. J. Comput. Phys. 227 (2008) 8841-8854.

[35] F. Hermeline, Approximation of diffusion operators with discontinuous tensor coefficients on distorted meshes. Comput. Methods Appl. Mech. Engrg. 192 (2003) 1939-1959.

[36] J. Hyman and M. Shashkov, Mimetic discretizations for Maxwell's equations and the equations of magnetic diffusion. Progress Electromagn. Res. 32 (2001) 89-121.

[37] J.M. Hyman, M.J. Shashkov and S. Steinberg, The numerical solution of diffusion problems in strongly heterogeneous nonisotropic materials. J. Comput. Phys. 132 (1997) 130-148.

[38] G. Lin, J. Liu and F. Sadre-Marandi, A comparative study on the weak Galerkin, discontinuous Galerkin, and mixed finite element methods. J. Comput. Appl. Math. 273 (2015) 346-362.

[39] K. Lipnikov and G. Manzini, A high-order mimetic method on unstructured polyhedral meshes for the diffusion equation. J. Comput. Phys. 227 (2014) 360-385.

[40] K. Lipnikov, G. Manzini, F. Brezzi and A. Buffa, The mimetic finite difference method for 3D magnetostatics fields problems. J. Comput. Phys. 230 (2011) 305-328.

[41] K. Lipnikov, G. Manzini and M. Shashkov, Mimetic finite difference method. J. Comput. Phys. B 257 (2014) $1163-1227$.

[42] K. Lipnikov, G. Manzini and D. Svyatskiy, Analysis of the monotonicity conditions in the mimetic finite difference method for elliptic problems. J. Comput. Phys. 230 (2011) 2620-2642.

[43] K.N. Lipnikov, J.D. Moulton, G. Manzini and M.J. Shashkov, The mimetic finite difference method for elliptic and parabolic problems with a staggered discretization of diffusion coefficient. Technical Report LA-UR-15-23755, Los Alamos National Laboratory, 2015. To appear in J. Comput. Phys. (2015). 
[44] K. Lipnikov, J.D. Moulton and D. Svyatskiy, A Multilevel Multiscale Mimetic $\left(\mathrm{M}^{3}\right)$ method for two-phase flows in porous media. J. Comp. Phys. 227 (2008) 6727-6753.

[45] K. Lipnikov, M. Shashkov and I. Yotov, Local flux mimetic finite difference methods. Numer. Math. 112 (2009) 115-152.

[46] G. Manzini, A. Russo and N. Sukumar, New perspectives on polygonal and polyhedral finite element methods. Math. Models Methods Appl. Sci. 24 (2014) 1665-1699.

[47] L. Margolin, M. Shashkov and P. Smolarkiewicz, A discrete operator calculus for finite difference approximations. Comput. Methods Appl. Mech. Engrg. 187 (2000) 365-383.

[48] A. Palha, P.P. Rebelo, R. Hiemstra, J. Kreeft and M. Gerritsma, Physics-compatible discretization techniques on single and dual grids, with application to the Poisson equation of volume forms. J. Comput. Phys. B 257 (2014) 1394-1422.

[49] N. Sukumar and E. Malsch, Recent advances in the construction of polygonal finite element interpolants. Arch. Comput. Methods Engrg. 13 (2006) 129-163.

[50] N. Sukumar and A. Tabarraei, Conforming polygonal finite elements. Int. J. Numer. Meth. Engrg. 61 (2004) $2045-2066$.

[51] E. Wachspress, A rational Finite Element Basis. Academic Press (1975).

[52] J. Wang and X. Ye, A weak Galerkin finite element method for second-order elliptic problems. J. Comput. Appl. Math. 241 (2013) 103-115.

[53] J. Wang and X. Ye, A weak Galerkin mixed finite element method for second-order elliptic problems. Math. Comput. 83 (2014) $2101-2126$. 